Western University

Scholarship@Western

Brain and Mind Institute Researchers'

Publications

Brain and Mind Institute

$10-1-2013$

\title{
The touchscreen operant platform for testing learning and memory in rats and mice.
}

\author{
Alexa E Horner \\ Christopher J Heath \\ Martha Hvoslef-Eide \\ Brianne A Kent \\ Chi Hun Kim
}

See next page for additional authors

Follow this and additional works at: https://ir.lib.uwo.ca/brainpub

Part of the Neurosciences Commons, and the Psychology Commons

Citation of this paper:

Horner, Alexa E; Heath, Christopher J; Hvoslef-Eide, Martha; Kent, Brianne A; Kim, Chi Hun; Nilsson, Simon R O; Alsiö, Johan; Oomen, Charlotte A; Holmes, Andrew; Saksida, Lisa M; and Bussey, Timothy J, "The touchscreen operant platform for testing learning and memory in rats and mice." (2013). Brain and Mind Institute Researchers' Publications. 99.

https://ir.lib.uwo.ca/brainpub/99 


\section{Authors}

Alexa E Horner, Christopher J Heath, Martha Hvoslef-Eide, Brianne A Kent, Chi Hun Kim, Simon R O Nilsson, Johan Alsiö, Charlotte A Oomen, Andrew Holmes, Lisa M Saksida, and Timothy J Bussey 


\title{
The touchscreen operant platform for testing learning and memory in rats and mice
}

\author{
Alexa E Horner ${ }^{1-3}$, Christopher J Heath ${ }^{2,3}$, Martha Hvoslef-Eide ${ }^{2,3}$, Brianne A Kent ${ }^{2,3}$, Chi Hun Kim²,3, \\ Simon R O Nilsson, ${ }^{2,3}$, Johan Alsiö ${ }^{2,3}$, Charlotte A Oomen ${ }^{2,3}$, Andrew Holmes ${ }^{4}$, Lisa M Saksida ${ }^{2,3}$ \& Timothy J Bussey 2,3
}

\begin{abstract}
${ }^{1}$ Synome Ltd., Babraham Research Campus, Cambridge, UK. ${ }^{2}$ Department of Psychology, University of Cambridge, Cambridge, UK. ${ }^{3}$ Behavioural and Clinical Neuroscience Institute, University of Cambridge, Cambridge, UK. ${ }^{4}$ Laboratory of Behavioral and Genomic Neuroscience, National Institute on Alcohol Abuse and Alcoholism (NIAAA), US National Institutes of Health, Bethesda, Maryland, USA. Correspondence should be addressed to A.E.H. (alexa.horner@cantab.net).
\end{abstract}

Published online 19 September 2013; doi:10.1038/nprot.2013.122

An increasingly popular method of assessing cognitive functions in rodents is the automated touchscreen platform, on which a number of different cognitive tests can be run in a manner very similar to touchscreen methods currently used to test human subjects. This methodology is low stress (using appetitive rather than aversive reinforcement), has high translational potential and lends itself to a high degree of standardization and throughput. Applications include the study of cognition in rodent models of psychiatric and neurodegenerative diseases (e.g., Alzheimer's disease, schizophrenia, Huntington's disease, frontotemporal dementia), as well as the characterization of the role of select brain regions, neurotransmitter systems and genes in rodents. This protocol describes how to perform four touchscreen assays of learning and memory: visual discrimination, object-location paired-associates learning, visuomotor conditional learning and autoshaping. It is accompanied by two further protocols (also published in this issue) that use the touchscreen platform to assess executive function, working memory and pattern separation.

\section{INTRODUCTION}

This protocol describes an automated touchscreen platform with which a remarkable diversity of cognitive functions may be tested in rodents. During more than 2 decades of research, a number of tasks have been designed and validated for the platform, each allowing the researcher to probe a unique set of functions ${ }^{1-5}$. Together these form a comprehensive battery of tasks, several of which may be used in concert by the researcher to elucidate a cognitive profile for a given rodent model; alternatively, they can be used more selectively to examine specific aspects of the cognitive repertoire in a hypothesis-driven manner.

The touchscreen platform has been used in a number of studies, in a variety of ways. First, putative rodent models of human conditions including Alzheimer's disease $e^{6,7}$, schizophrenia ${ }^{8-10}$, Huntington's disease ${ }^{11}$, frontotemporal dementia (A.E.H., B.A.K., T.J.B. and L.M.S., unpublished data), aging ${ }^{12}$, exposure to stress ${ }^{13}$ and substance abuse ${ }^{14}$ have been studied. Notably, we recently demonstrated the utility of this platform for parallel cognitive testing of humans with schizophrenia and a putative mouse model of the disease (discs, large homolog 2 (Dlg2) knockout) sharing a similar genetic basis ${ }^{8}$. Second, these tasks have been used to investigate the neural underpinnings of a number of different cognitive functions, targeting the rhinal ${ }^{15-18}$, medial and ventromedial prefrontal ${ }^{13,19-21}$, anterior and posterior cingulate ${ }^{22-26}$, medial frontal ${ }^{22,23}$, orbitofrontal ${ }^{13,27,28}$, infralimbic ${ }^{28}$ and prelimbic ${ }^{29}$ cortices. In the striatum, studies of dorsolateral and dorsomedial areas $13,20,21$ and the nucleus accumbens $22,25,26,30,31$ have been performed. Roles for a number of other brain regions, including the amygdala ${ }^{25,32}$, distinct thalamic nuclei ${ }^{29}$, the subthalamic nucleus $^{33}$, the fornix ${ }^{17,34}$, subiculum ${ }^{32}$, hippocampus $27,35-41$, pedunculopontine tegmental nucleus ${ }^{42}$, medial septal/vertical limb of diagonal band (cholinergic neurons ${ }^{43}$ and nucleus basalis magnocellularis (cholinergic neurons) ${ }^{44}$ have also been identified in a number of tasks. Third, the efficacy of systemic pharmacological agents has been studied, using compounds active on the cholinergic ${ }^{7,45-48}$, dopaminergic ${ }^{14,31,48-50}$, glutamatergic ${ }^{9,48}$ and serotonergic ${ }^{50-53}$ systems. Fourth, the function of specific genes $8,51,54,55$, receptors ${ }^{56}$, receptor subunits ${ }^{57-59}$ and structural plasticity processes, such as adult hippocampal neurogenesis ${ }^{12,60 \text {, }}$ have been assessed.

\section{Advantages and disadvantages of the touchscreen platform} The advantages of the touchscreen platform have been discussed in detail elsewhere ${ }^{1-3}$. Briefly, this platform offers the potential for a high degree of standardization, minimal experimenter involvement and high translational potential (e.g., similarity to human CANTAB (Cambridge neuropsychological test automated battery) tests). It includes assays of various neuropsychological constructs, including attention and cognitive flexibility, and it uses appetitive rather than aversive motivation. One obvious advantage of using computer-generated visual stimuli is that the perceptual features (size, shape, contrast, luminance and so on) and similarities of the stimuli can be easily manipulated ${ }^{3,61}$. Furthermore, in object-based tasks in which the objects are displayed in different locations on the touchscreen, there is no potential for the use of odor cues, unlike some (dry) maze tasks, which can modify results. The platform also lends itself to applications that allow for the measurement of brain functions in vivo as animals perform a task (for example, via single-unit neuronal recordings $s^{62}$ ). There is potential for the incorporation of other powerful methodologies (e.g., optogenetics) into the touchscreen platform. Although we focus on rodents in this article, touchscreens have been used with pigeons and nonhuman primates, as well as with mice and rats $2,63-68$.

It is worth noting that although automated methods such as the touchscreen platform reduce experimenter effort, the tasks can take many more sessions to run than equivalent tests using, e.g., odors. However, because tests on large numbers of animals $(>20)$ can be run in parallel, experiments can often be completed in the same number of days (or fewer) as they can with 'hand-testing' methods in which an experimenter tests one animal at a time. 
Furthermore, although the hand tester is working on one experiment all day, the experimenter with a number of automated units can work on several experiments. Of course, to achieve this high throughput, one needs the apparatus, which means a larger initial financial outlay than is required for most hand-testing methods. Again, however, if one considers all factors, such as salaries and person-hours spent on experiments, and the fact that such apparatus can be used for many years before needing to be replaced, in the long run automation may actually be less expensive than hand-testing alternatives.

Another potential limitation is that the use of visual stimuli precludes the use of certain subjects, such as mice with genetic alterations that cause rapid retinal degeneration. (Albino rats, however, seem to have sufficient acuity to perform as well in the touchscreen as pigmented rats $^{3}$.) In addition, as with most appetitive, operant paradigms, the use of food reward may introduce possible problems; for example, an experimental treatment may affect appetite or interact with the physiological effects of food restriction. These limitations should be kept in mind, although we do believe that all things considered, the advantages conferred by avoiding aversive stimuli far outweigh the disadvantages of using appetitive stimuli. Touchscreen tasks require intact motoric function such that subjects are able to traverse the testing chamber, respond to the screen and collect and consume the food reward. Again, however, these demands are much lower than many currently used behavioral paradigms. Importantly, the impact of most of these potential changes can be assessed by taking a battery approach, by running appropriate control experiments and/or inspecting relevant dependent variables such as trial omissions and/or reaction times to respond or to collect the reward. If one takes a battery approach, testing the effect of a given experimental manipulation on several tasks, then the tasks can act as mutual controls by virtue of the fact that they involve the same types of apparatus, stimuli, responses and reinforcement ${ }^{1}$; comparisons can be made confidently between tasks in the battery because such variations are minimal. For example, if an animal performs poorly in object-location paired-associates learning (which theoretically requires cognitive functions including visual discrimination and learning of object-location associations; discussed further below), but well in visual discrimination (which requires visual discrimination learning; discussed further below), it would be reasonable to conclude that the former impairment is not due to a general problem in perceptually discriminating images. Similarly, we have found that muscarinic M2 receptor-knockout mice are impaired in object-location paired-associates learning, but they actually demonstrate improved attention in the five-choice serial reaction time (5-CSRT) task (Romberg, C. et al., unpublished data) making it very unlikely that the former impairment is due to an attentional deficit.

Finally, for researchers for whom the ethological validity of a method is important, rodents using touchscreens may not be the method of choice. However, we note that the behavior in the touchscreen is built on the natural tendency of rodents to approach and explore novelty in the environment; the exploration is detected by the touchscreen, and the animal learns, again quite naturally, the consequences of exploring certain stimuli. In this sense, the method is no less ethologically valid than having rodents swim in an artificial pool in a laboratory setting, or other commonly used laboratory methods. In any case, we see the touchscreen method as complementing rather than replacing other methods such as foraging paradigms.

\section{Assessing learning and memory}

This protocol describes four tasks that may be used to assess aspects of learning and memory. The first three of these rely primarily on appetitively motivated instrumental learning, and are preceded by 'pretraining', in which subjects must learn to make instrumental responses in the touchscreen apparatus. Visual discrimination is a relatively simple task, in which subjects must learn to consistently respond to one of two visual stimuli. In object-location paired-associates learning, the correct stimulus is identified by the conjunction of a visual stimulus and its location on the touchscreen. In visuomotor conditional learning (VMCL), the correct response (left or right) depends on which conditional visual stimulus is presented. Autoshaping is unique in the battery, primarily testing Pavlovian stimulus-reinforcer learning. Two accompanying protocols discuss additional tasks that may be used to assess working memory and pattern separation 4 (trial-unique non-matching-to-location (TUNL) and location discrimination (LD)) and executive function ${ }^{5}$ (reversal, extinction and the 5-CSRT task). Other tasks that will further expand the range of the battery are constantly in development.

\section{Visual discrimination (Step 10A)}

Learning to discriminate between environmental stimuli is essential in order to successfully shape decisions and adaptively guide behavior. Understanding the neural mechanisms supporting discrimination learning is of major interest to cognitive neuroscience, and it may have implications for delineating the pathophysiology of cognitive impairments in neuropsychiatric disorders such as schizophrenia and Parkinson's disease. Basic preclinical research in animals is key to this work, and various methods for testing discrimination learning have been developed, including touchscreen-based systems in nonhuman primates ${ }^{69}$. In addition to the basic pairwise discrimination procedure, certain variations have also been developed, including multidimensional ${ }^{70}$ (to test attentional set-shifting), concurrent ${ }^{23}$ and conditional (see VMCL) discriminations, as well as transverse patterning 34 (to test configural learning).

Initial studies using a touchscreen discrimination procedure were published almost 20 years ago; they used a configuration that included a monitor, off-the-shelf operant hardware and customized software ${ }^{2}$ (see also ref. 71). Briefly, the procedure entails simultaneous presentation of two stimuli, and the measurement, over multitrial sessions, of the animal's ability to reliably touch the stimulus designated the conditioned stimulus (CS) ${ }^{+}$ (rewarded) in favor of the other stimulus ( $\mathrm{CS}^{-}$, nonrewarded). Discrimination learning requires at least two processes: learning to perceptually discriminate the stimuli, and learning which of the two stimuli is associated with reward. It also provides the basis for testing reversal learning ${ }^{5}$, in which the stimulus-reward contingencies acquired during discrimination are reversed.

The task has been used to investigate a variety of questions concerning the neural basis and pharmacological modulation of visual discrimination learning. These include testing the effects of drug treatments including psychotomimetics and putative cognitive enhancers $9,13,18,47$; gene mutations, particularly of glutamate signaling molecules $8,10,51,55,57-59$; discrete brain lesions ${ }^{13,23,28,72-74}$; and environmental manipulations such as exposure to stress ${ }^{13}$. 


\section{Object-location paired-associates learning (Step 10B)}

The formation of an association between two individually neutral stimuli, named paired-associate learning (PAL), has been extensively studied in humans using a variety of modalities (verbal, visual, locations). Although PAL has traditionally been assessed using pairings of words tested by cued recall, the human CANTAB PAL task ${ }^{75}$ does not rely on verbal stimuli and thus provides a version of PAL that is more amenable to modeling in animals. The computerized PAL task requires the subject to form an association between a visual stimulus and its location on a screen, demonstrated under cued-recall conditions. Over more than 2 decades, CANTAB PAL has been validated as sensitive to detecting deficits in a range of conditions such as schizophrenia $^{76-78}$, Huntington's disease ${ }^{79}$, Parkinson's disease ${ }^{75}$, major depressive disorder ${ }^{80}$, unipolar and bipolar mood disorders ${ }^{81}$ and Alzheimer's disease ${ }^{75,79,82-86}$.

Given the profile of neuropsychiatric disorders to which object-location learning is sensitive, it is not surprising to find that encoding and retrieval of object-location associations has been linked to hippocampal and prefrontal cortical function ${ }^{87-89}$. Importantly, the same areas have been implicated in the rodent touchscreen object-location paired-associates learning task developed by Talpos and colleagues ${ }^{41,90}$, in which the animal is required to learn three individual object-location associations. Each visual stimulus (object) is correct in a unique location, which stays stable throughout training. On each trial, two different objects are presented, one in its correct location and the other in an incorrect location. The third location remains blank. The rodent task differs from that of CANTAB PAL in that the stimuli are not trial unique, and the task does not feature a delay. Importantly, however, the requirement to use both object and location information to solve the task is maintained. Indeed, assessment of paired-associates learning using CANTAB PAL in people with DLG2 mutations produced a similar phenotypic profile to that observed by using the rodent object-location paired-associates learning task ${ }^{8}$ with Dlg2-knockout mice, indicating the translational potential of the paradigm. We note that, in this task, the animal can approach locations on the screen from many different angles, which is in contrast to the behavior that we see in, e.g., the VMCL task.

Pharmacological manipulation of the rodent object-location paired-associates learning task indicates that both facilitation and disruption of performance is possible. Antagonism of NMDA or AMPA receptors in the hippocampus impairs performance in rats, but leaves accuracy unaltered for a similar control task, which may be solved by visuomotor conditional learning as opposed to the formation of object-location associations ${ }^{41}$. Systemic pharmacological manipulations in mice have further implicated cholinergic muscarinic receptors in performance of the task, with a facilitation observed in wild-type animals using donepezil ${ }^{45}$. Knockout of muscarinic M2 but not muscarinic M1 receptors impairs acquisition of the task ${ }^{56}$ (Romberg, C. et al., unpublished results). Task performance is sensitive to amphetamine but not to PCP, ketamine or LSD ${ }^{91}$. Thus, the task offers an automated and sensitive measure of rodent object-location paired-associates learning and performance, which has translational potential.

\section{VMCL (Step 10C)}

In VMCL, animals learn a conditional rule of the type 'If visual stimulus $A$ is presented, make motor response $\mathrm{X}$; if visual stimulus B is presented, make motor response Y'. There has been considerable interest in such visuomotor mapping in primates ${ }^{92}$. Generally, it appears that across monkeys and rodents, hippocampal damage does not consistently produce impairments in such tasks, although the hippocampal system can become involved when mappings are acquired rapidly or involve object-location rather than visuomotor associations ${ }^{93,94}$. Rodent VMCL in operant chambers requires discrete left-right responses and thus probably involves visuomotor associations, which are likely to require stimulus-response habit learning; as would therefore be expected, the task is more sensitive to damage in the striatum than in the hippocampus ${ }^{95,96}$. The VMCL task in the touchscreen is indeed designed to maximize stimulus-response learning and minimize other cognitive demands. Thus, the discrimination is chosen to be an easy one (in practice probably solved via light-dark discrimination), to reduce perceptual demands. Furthermore, a 'limited hold' (time limit) for responding promotes the same rapid headturn-and-nose-poke motor response on each trial, encouraging a visuomotor strategy and limiting the extent to which subjects can move away from the screen and reapproach the choice stimuli from different angles, which might promote alternative learning strategies. Touchscreen VMCL does not require medial prefrontal cortex, anterior cingulate cortex, hippocampus, perirhinal cortex, anterior thalamus or mediodorsal thalamus, but it does depend on posterior cingulate cortex (late in learning only) $23,74,97$, thus conferring the specificity needed to dissociate function as part of a touchscreen test battery ${ }^{23}$. As in pairwise visual discrimination learning, the task can also be reversed to engage a different set of brain regions $22,43,74$. The VMCL task may be particularly relevant to Huntington's and Parkinson's disease, in which cognitive impairments include deficits in habit learning ${ }^{98,99}$.

\section{Autoshaping (Step 10D)}

The autoshaping task assesses Pavlovian approach learning. It capitalizes on the process of 'autoshaping', which was first observed in experiments in which pigeons came to reliably peck at an illuminated key (CS) presented immediately before delivery of grain at a separate location ${ }^{100}$, and has been reported in many species $^{8,101-106}$. It is considered to rely on Pavlovian, as opposed to instrumental, associations ${ }^{22}$. Although a behavioral chamber equipped with levers can be used to assess rodent autoshaping 107 , this protocol details the use of a touchscreen system as originally described by Bussey et al. ${ }^{22}$.

Autoshaping is a discriminative conditioning procedure, in which a stimulus is presented on either the left or right side of the touchscreen, with one side defined as $\mathrm{CS}^{+}$(rewarded CS) and the other as $\mathrm{CS}^{-}$(nonrewarded CS). Reward is delivered upon termination of the $\mathrm{CS}^{+}$but not the $\mathrm{CS}^{-}$. With repeated presentations, rodents increase $\mathrm{CS}^{+}$approaches and decrease $\mathrm{CS}^{-}$approaches, indicating that the predictive relationship between $\mathrm{CS}^{+}$presentation and reward delivery has been learned ${ }^{22}$. To demonstrate the Pavlovian nature of the association, a reward omission procedure 22,108 can be implemented, in which $\mathrm{CS}^{+}$approaches cause reward to be withheld. Under this altered contingency, animals continue to respond to the $\mathrm{CS}^{+}$, which is consistent with a Pavlovian CS-UR association 22,108 .

This task requires minimal pretraining and animals quickly develop the necessary stimulus discrimination, making it relatively rapid to complete. Therefore, it has been used extensively 
to characterize the neurobiological mechanisms underlying Pavlovian learning in conditions in which the effects of instrumental learning mechanisms on performance should be minimized ${ }^{26}$. In particular, studies of rodent autoshaping after disruption of defined brain regions have identified critical roles for the nucleus accumbens core ${ }^{25,26,109}$, anterior cingulate cortex ${ }^{22,24,25}$ and the projections between them $22,25,26,110$. A number of other structures, including the orbitofrontal corte ${ }^{28}$, central nucleus of the amygdala ${ }^{32}$, pedunculopontine tegmental nucleus ${ }^{42}$ and subthalamic nucleus ${ }^{33}$ are also required. Lesions of the hippocampus appear to enhance autoshaping acquisition ${ }^{36}$. The anatomical specificity of this task is striking, as the disruption of other closely related brain regions, such as the basolateral nucleus of the amygdala ${ }^{32}$, nucleus accumbens shell ${ }^{26}$, dorsal striatum ${ }^{20,21}$, posterior cingulate cortex ${ }^{22}$, medial prefrontal cortex $20-22$ and infralimbic $\operatorname{cortex}^{28}$ have no effect. This task is also sensitive to systemic administration of a number of pharmacological agents, including typical and atypical antipsychotics ${ }^{111}$ and apomorphine $^{31}$. Central administration of a variety of neurotransmitter receptor antagonists has indicated that functional glutamatergic and dopaminergic accumbens signaling is required ${ }^{30,107}$. These features make the task valuable in furthering the understanding of stimulus-reinforcer learning generally, and particularly if the reinforcer is maladaptive, as in drug addiction 25,107 . It has also been suggested that aspects of the task can model impulsive and perseverative responding 33,53 . The strong dependence of the task on dopaminergic and glutamatergic signaling may also be of value in studies of conditions in which these are disrupted, such as schizophrenia ${ }^{112,113}$, with potential for relatively rapid screening of novel rodent models or therapeutics. Furthermore, one could conceivably monitor magazine entry during stimulus presentation to measure goal-tracking in addition to sign-tracking behavior ${ }^{114}$. We are currently exploring this possibility, which may prove particularly useful for models of neuropsychiatric disease. For example, Danna and Elmer ${ }^{111}$ found that the atypical antipsychotic olanzapine and typical antipsychotic haloperidol disrupted the conditioned approach to a reward-predictive cue (sign tracking), but neither drug disrupted the conditioned approach to the reward (goal tracking). Furthermore, in the context of drug addiction, it has also been shown that differences in sign tracking and goal tracking can reflect underlying differences in the dopamine system ${ }^{114}$ and are linked to the responsiveness to drugs of abuse ${ }^{115}$.

\section{Experimental design}

General considerations. Task-specific experimental details are described below in sections dedicated to each task. Unless stated otherwise, the tasks are described here in the way that we presently conduct (or intend to conduct) them. In this first section, some general principles, advice and alternatives are discussed.

-Apparatus type. We use two types of touchscreen apparatus: inhouse assembled apparatus and apparatus that is commercially available from Campden Instruments. Both are described in MATERIALS. The majority of tasks presented here have been performed in both.

- House light. Our current standard procedure is to have the house light off during stimulus presentation and inter-trial intervals (and on for 'time-out' periods), but the majority of tasks have also been performed with the house light on, and we do not have conclusive evidence that these variations affect task performance.

- Reward. Two types of reward are typically used: liquid or solid (see MATERIALS). Pellets seem to work well for rats. We use either liquid or solid for mice; liquid rewards may be a better choice in some cases, e.g., when you are using manipulations that result in motoric changes that could affect chewing, cause dry mouth or reduce motivation.

-Inter-trial intervals (ITIs). The ITI in the tasks presented in this paper is $20 \mathrm{~s}$ (except for autoshaping). Although shorter ITIs are frequently used, particularly with mice (e.g., $15 \mathrm{~s}$ (refs. 13,51), $5 \mathrm{~s}$ (refs. 19,49,57,70)), longer ITIs may facilitate learning ${ }^{3}$.

- 'Free' initial reward delivery. In the majority of touchscreen tasks (excluding stages 1-3 of pretraining, autoshaping and extinction), a free reward is delivered (e.g., one reward pellet or $20 \mu \mathrm{l}$ of milkshake) at the start of each session to prime responding and encourage initiation of the first trial. This may be delivered manually before the start of the session, or automatically at the start of the session by the software program.

- Correction trials $(C T s)$. When the subject makes an incorrect response, the next trial initiated will be a CT (in the majority of tasks; see task-specific Experimental design), in which the same stimulus or stimuli are represented in the same location(s). CTs do not count toward the session trial limit, or the main accuracy score (see Data analysis below). There is usually no limit on the number of CTs that can be given consecutively, but once the subject responds correctly the correction procedure ends. The purpose of CTs is to counteract side and stimulus biases, and to ensure that subjects receive a consistent number of rewards per session despite their performance on noncorrection trials.

Data analysis. There are several performance measures common to the majority of touchscreen tasks. The measures recorded for each animal in each session of these tasks include the following: number of responses to blank/correct/incorrect stimuli (for correction and noncorrection trials separately), total number of trials and CTs completed, correct/incorrect response latency and reward retrieval latency. From these, the following measures may be calculated for each phase of an experiment:

- Percentage accuracy $=(100 \times($ correct responses $) /($ correct + incorrect responses)), which is often plotted as a function of session, i.e., an acquisition curve. Note that this measure does not include CTs.

- The number of sessions/trials/errors (incorrect responses in noncorrection trials) to attain a specified performance criterion. - Average latency to make a correct/incorrect response after the presentation of stimuli (also termed reaction time). Note that data from CTs are usually not included in this measure.

- Average latency to collect reward after a correct response is made (also termed magazine latency). Latencies to respond and collect reward (usually in noncorrection trials only) can reveal perturbations in motivation, motoric function, speed/error trade-off and so on ${ }^{116}$.

- In cases in which bias toward a specific location or stimulus may affect responses (e.g., visual discrimination, VMCL), percentage of bias can be calculated, e.g., for the first session. This is the number of trials in which the subject responds to a particular location/stimulus, expressed as a percentage of all trials. In cases in which a treatment affects innate stimulus bias, assessing the rate of task acquisition will be problematic, as the treatment 
and control groups will not be at similar performance levels (i.e., chance) at the outset of the experiment.

- A perseveration score (also termed perseveration index) may be calculated to assess the extent to which subjects perseverate in responding to the incorrect location/stimulus during CTs after an incorrect response, corrected for the number of initial incorrect responses (on first presentation trials). This may be expressed as the average number of CTs per incorrect response.

- Screen touches during ITI/time-out may be calculated, and might provide an additional measure of perseveration or motoric activity.

If performance is expected to vary within session, for example, after drug administration, it may be useful to analyze the abovementioned measures in bins of, e.g., ten trials.

Experimental manipulations. In all of the tasks described here, the specific research question and experimental manipulation determine the behavioral procedure. For clarity, we will describe four possible treatment scenarios. In case 1, the subject receives treatment before the onset of the experiment (e.g., constitutive transgenic or knockout models, developmental manipulations). In case 2, the subject receives treatment before task acquisition, but after pretraining (e.g., subchronic drug treatment, neurotoxic lesions). In case 3, the subject receives treatment after acquisition to assess the effects on asymptotic performance level, or on postacquisition behavioral challenges, by using a between-subject design (e.g., neurotoxic lesions, subchronic drug treatment). In case 4, the subject receives a transient manipulation at asymptotic performance level, or during postacquisition behavioral challenges, that can be performed within-subject (e.g., systemic pharmacological or infusion procedures). We will refer to these cases as appropriate in our protocols.

When postacquisition manipulations are of interest (including cases 3 and 4, and postacquisition behavioral challenges), there are several options for the point at which animals should be advanced from acquisition training. First, a group of animals may all be tested for a prespecified number of acquisition sessions, and then all advance to the postacquisition manipulation regardless of performance level. An advantage is that all animals in the group will be synchronized (i.e., the manipulation will begin for all animals on the same day), which minimizes variability due to extraneous factors, is ideal for pharmacological studies (because injections (whether vehicle or drug for a given animal) may be conducted on the same day(s) for all animals) and enables decisions (e.g., concerning the number of days for which to run a manipulation) to be made ad hoc on the basis of the group's mean performance level. This is also particularly important when subjects must be of the same age at the start of each testing phase (for example, when testing a progressive disease model). However, there will be some variation in the performance levels of the animals at the end of training, and some may not have acquired the initial task to a sufficient baseline level from which to assess alterations in performance due to a manipulation.

Second, a group of animals may be trained until all animals in the group have reached a performance criterion. However, although this means that the group will be synchronized and will have the same number of training days before the postacquisition manipulation (allowing for an acquisition curve to be plotted), some animals will be overtrained.
Third, each animal in the group may be trained until it reaches criterion, and then individually advanced to the manipulation of interest. Although this avoids overtraining and variations in performance level, the group is not synchronized.

We suggest a fourth option: each animal in the group is trained daily (at least $5 \mathrm{~d}$ per week) until it reaches criterion, upon which it is 'rested' without daily training (although food restriction continues). Subjects on rest are usually given one or two 'reminder' training sessions per week unless it is anticipated that all subjects will reach criterion within a few days of each other. If an animal's performance falls below criterion in a reminder session, that animal is trained daily until criterion is reattained. When all animals have reached criterion (at least) once, they are rebaselined as a group (i.e., all animals are trained daily). Postacquisition manipulations may begin when performance of all subjects has been stable at criterion for at least $2 \mathrm{~d}$. Although subjects receive a different number of training days, precluding plotting of a complete acquisition curve, the animals are synchronized, with minimal variation in their performance levels, and overtraining is minimized.

Flexible battery approach. The tasks presented in this set of articles $^{4,5}$ form part of a flexible battery ${ }^{1}$, meaning that the tasks and task order used can be tailored by the researcher to address specific hypotheses and research requirements. Although this is not suitable for all types of manipulation (e.g., progressive disease models, drug studies), we suggest using a battery approach to elucidate a cognitive profile where appropriate. This approach is particularly suitable when there are no specific hypotheses regarding the domains of cognition that will be affected by a manipulation. Here a single group of animals is tested on multiple tasks from the battery, as well as probes if appropriate (see post-training manipulations in task-specific procedures). In comparison with the other extreme of testing a naive cohort on each task, this battery approach requires fewer animals and is more efficient (full pretraining is only required before the first task), although further research is necessary to explore order effects and the potential for negative or positive transfer between different task combinations. We have settled on two mini-batteries of tasks for mice, which comprise the six most commonly used tasks available. (Autoshaping is usually tested in a dedicated cohort.) One cohort is tested upon visual discrimination, reversal, objectlocation paired-associates learning and extinction, and a second is tested on 5-CSRT and $\mathrm{LD}^{8}$. By using these sequences, we have not observed marked transfer effects in control or experimental groups of mice. However, order effects may only be conclusively ruled out by retesting naive animals on the task in question. Order effects are an important consideration for all cognitive and behavioral testing of rodents in which the same animals are tested on more than one task.

Pretraining. All tasks in the touchscreen battery are motivated by food reward, and the majority of the tasks require instrumental responses to the touchscreen. Therefore, to provide sufficient motivation, animals are subject to mild food restriction before task training. Pretraining normally consists of five stages, followed by training specific to the task. As described previously6-8,12,51,63, these gradually shape the screen-touching behavior required by all of the instrumental touchscreen tasks (an exception is the 
PROTOCOL

TABLE 1 | Mask and stimulus dimensions.

\begin{tabular}{|c|c|c|c|c|c|}
\hline Rat/2/In-house & VD & $15.0 \times 9.2$ & 2.5 & 12.5 & $9.0 \times 9.0$ \\
\hline Mouse/2/In-house & VD & $7.0 \times 5.3$ & 0.5 & 1.5 & $4.0 \times 4.0$ \\
\hline Rat/2/Campden & Auto & $30.0 \times 6.4$ & 9.6 & 0.0 & $25.8 \times 5.7$ \\
\hline Mouse/2/Campden & Auto & $17.5 \times 8.2$ & 7.5 & 0.0 & $15.7 \times 7.3$ \\
\hline Rat/3/In-house & $\mathrm{PAL}, \mathrm{VMCL}$ & $15.1 \times 6.0$ & 1.5 & 12.5 & $6.0 \times 5.7$ \\
\hline
\end{tabular}

Auto, autoshaping; PAL, object-location paired-associates learning; VD, visual discrimination; VMCL, visuomotor conditional learning.

All dimensions are approximate, and given as height $\times$ width, in $\mathrm{cm}$. Window gap is the horizontal distance between windows. Stimuli are always positioned centrally on the horizontal axis of the screen. Floor gap is the vertical distance between the bottom of the stimulus window and the floor.

aNew rat paired-associates learning stimuli (Fig. 5b) are $10.0 \times 6.0$.

Pavlovian autoshaping task). The number and size of response windows and the size and type of visual stimuli used during pretraining depend on the task that the subject is to be trained on subsequently. If that task uses plain white square stimuli (including VMCL), the pretraining stimulus is usually a plain white square. For other tasks that use discriminative stimuli only (including object-location paired-associates learning, visual discrimination), pretraining stimuli are from a library of 40 varied black and white shapes, none of which substantially resemble the stimuli used in these tasks. The rationale for this procedure is that generalization between the training and task-specific stimuli should be minimized. The number and size of response windows and stimuli for tasks in the paper can be found in Table 1. We note that rats are typically given the opportunity to complete more trials per session than mice (e.g., 100 as opposed to 30 during pretraining). Rats readily complete a greater number of trials per session than mice, perhaps because the mouse:rat body mass ratio is smaller than the mouse:rat reward pellet size ratio (14 mg:45 mg).

After the introduction of mild food restriction, animals are habituated to the chambers and to food rewards for at least two daily sessions (see PROCEDURE; stage 1). In stage 2 (Fig. 1), the relationship between offset of a visual stimulus on the screen and delivery of reward is introduced. A stimulus is presented in one of the response windows (with the same location not used more than three times consecutively). If it is not touched, offset occurs after $30 \mathrm{~s}$ and a reward is delivered, along with illumination of the magazine and a tone (e.g., 1 s, $3 \mathrm{kHz}$; conditioned reinforcer). Touches to stimuli on the screen are encouraged with immediate offset, a triple reward delivery, tone and magazine illumination. When the animal enters the magazine to retrieve the reward, the magazine light is turned off and an ITI begins, after which the next trial is automatically initiated.

Stage 3 (Fig. 1) is similar to stage 2, but stimulus offset is dependent on the subject touching it. A stimulus is presented in one of the response windows, and remains there until it is touched, upon which the stimulus disappears and a reward is delivered accompanied by a tone and magazine illumination. When the animal enters the magazine to retrieve the reward, the magazine light is turned off and an ITI begins, after which the next trial begins automatically.

Stage 4 (Fig. 1) is similar to stage 3, but subjects are required to trigger stimulus presentation, referred to as trial initiation. The session begins with a free reward delivery and magazine illumination, indicating that a trial may be initiated. When the animal's nose pokes into the magazine, the magazine light is extinguished and a click sounds $(0.2 \mathrm{~s})$, and when the animal withdraws from the magazine, stimuli are presented on the screen. Initiation is also required after each ITI.

Stage 5 (Fig. 1) is similar to stage 4, but subjects are discouraged from touching blank response windows during stimulus presentation, with stimulus removal and a 5-s time-out period in which the house light is inverted. After the time-out, an ITI begins, after which the next trial can be initiated. However, in pretraining preceding the instrumental tasks in this paper, a CT is given instead of a new trial (see General considerations above). This stage also serves to introduce the subject to the cue signaling incorrect responses (the time out). By the end of pretraining, subjects should be completing a sufficient number of trials per session (as specified in PROCEDURE), to promote completion of sessions in the subsequent task.

Analysis of pretraining performance is minimal. The number of sessions required to complete each phase of pretraining, or the overall number of sessions required to complete pretraining, may be analyzed ${ }^{6-9,12,51,52,55,57,59,63}$. In addition, if you use an apparatus that permits assessment of activity in the chambers, measurements pertaining to this (e.g., number of beam breaks per half hour) may be analyzed as well.

Visual discrimination (Step 10A). This protocol is based on recent mouse and rat publications $6,8,13,47,58$ with minimal 
Figure 1 | Flowchart overview of pretraining stages 2-5. Stage 2: a visual stimulus is presented in one of the response windows. If it is not touched, stimulus offset occurs after $30 \mathrm{~s}$ and a reward is delivered. If it is touched, the offset is immediate and a triple reward is delivered. After reward collection and an ITI period, the next stimulus is presented in a new trial. Stage 3: proceeds as in stage 2, but the stimulus remains on the touchscreen until it is touched. Stage 4: proceeds as in stage 3, but the animal must enter and exit the magazine after the ITI to initiate the next trial. Stage 5: proceeds as in Stage 4, but touches to blank response windows (when there is a stimulus on the screen) are discouraged with a time-out. After this and the ITI, the next trial may be initiated, but in pretraining for the majority of tasks this is a CT in which the previous stimulus is represented rather than a new trial. Note that CTs are not given in stage 5 of pretraining for LD and 5-CSRT. The labels in italics indicate steps in which the animal is required to perform an action.

changes. A visual discrimination session (Fig. 2) begins with a free reward delivery and magazine light illumination, indicating that a trial may be initiated (as in pretraining). After initiation, two stimuli $\left(\mathrm{CS}^{+}\right.$and $\left.\mathrm{CS}^{-}\right)$appear in the two response windows. The locations of the $\mathrm{CS}^{+}$and $\mathrm{CS}^{-}$are pseudorandom, with the stimuli not displayed in the same locations for more than three consecutive trials (excluding CTs). The reward contingencies may be counterbalanced, such that for some animals a given stimulus will be $\mathrm{CS}^{+}$and the other $\mathrm{CS}^{-}$, whereas for other animals the reverse will be true. If the animal touches the $\mathrm{CS}^{+}$(correct), the stimuli are removed and a reward is delivered along with illumination of the magazine light

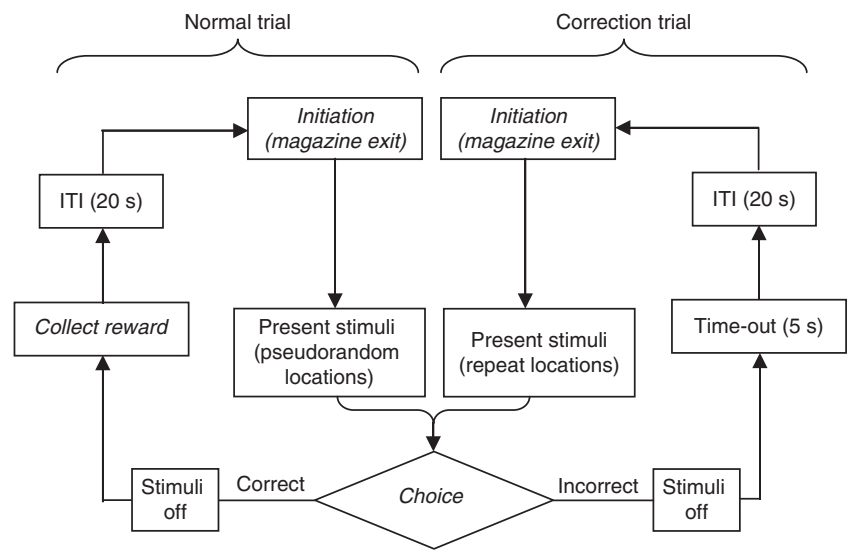

Figure 2 | Flowchart overview of the visual discrimination task. After initiation, a pair of stimuli $\left(\mathrm{CS}^{+}, \mathrm{CS}^{-}\right)$is presented on the screen in pseudorandom locations. Correct responses (to $\mathrm{CS}^{+}$) are rewarded, and after reward collection and an ITI a new trial may be initiated. Incorrect responses (to $\mathrm{CS}^{-}$) are discouraged with a time-out, and then after an ITI and initiation, the previous trial type is represented (a CT). The CT loop will continue until a correct response is made. The labels in italics indicate steps in which the animal is required to perform an action. and a tone $(1 \mathrm{~s}, 3 \mathrm{kHz})$. When the animal enters the magazine to retrieve the reward, the magazine light is turned off and an ITI begins, after which the magazine is again illuminated to indicate that a new trial may be initiated. If the animal touches the $\mathrm{CS}^{-}$ (incorrect), the two stimuli are removed and the house light is inverted for a 5-s time-out period, after which an ITI begins, and then the next trial may be initiated. However, instead of a new trial (as would be presented after a correct response), a CT is given (see General considerations).

Various training stimuli may be used (Fig. 3). For rats, the 'spider-plane' pair (Fig. 3a) is typically used 3,47 . For mice, the 'marble-fan' (Fig. 3b) pair (used in the majority of previous publications $6,8,11,13,55,59,63)$ is typically used in our purpose-built apparatus. Both rats and mice are also able to discriminate complex photographic stimuli ${ }^{3,8,56,59}$ (e.g., Fig. 3d). We have also recently developed 'lines-grid' (Fig. 3c and Supplementary Video 1) stimuli in the Campden apparatus, which optimize the rate of acquisition but minimize stimulus bias in that apparatus. Depending on the hypotheses under investigation, morphed stimuli (Fig. 3e) with overlapping features $47,48,56$ can be used to increase the difficulty of the discrimination, usually as post-training behavioral challenges once subjects have acquired the initial discrimination. These may reduce possible ceiling effects and may thereby increase the potential for detecting experimentally induced improvements ${ }^{47}$. Tests under various difficulty levels also allow examination of interaction between task difficulty and the experimental manipulation ${ }^{48}$. We note that there are several examples in the literature of alternative visual discrimination stimuli, apparatus and experimental designs $35,46,48,61,65,71,117,118$. Another option is 
a
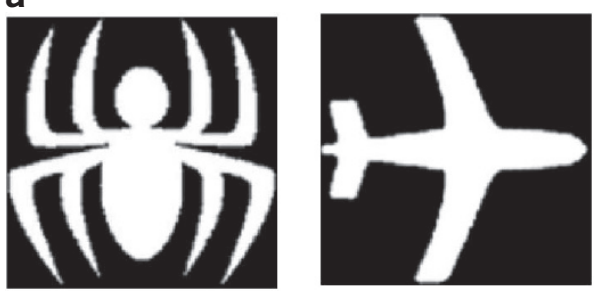

b

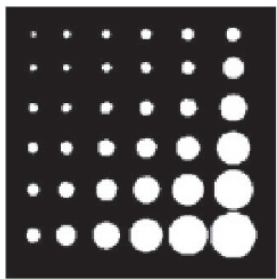

C

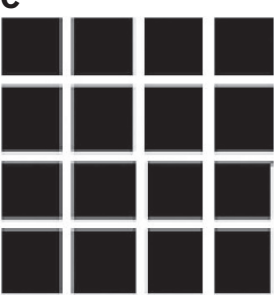

d

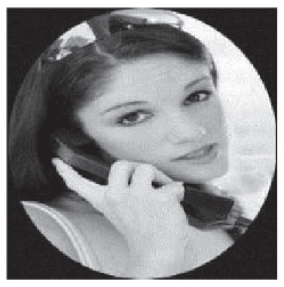

e
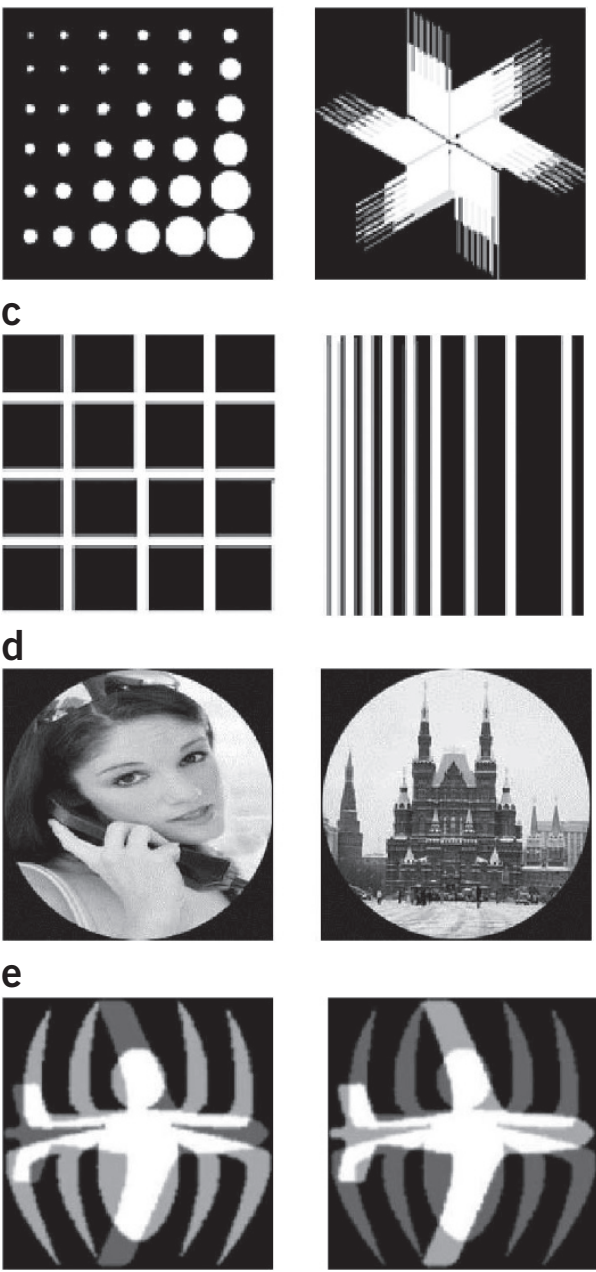

Figure 3 | Stimulus pairs recommended for use in visual discrimination and reversal learning. (a) 'Spider-plane' (reproduced from ref. 3 with permission). (b) 'Marble-fan' (reproduced from ref. 63 with permission not required).

(c) 'Grid-lines.' (d) Photographic 'face-building' (reproduced from ref. 3 with permission). (e) Morphed 'spider-plane' (60\%/40\%; reprinted from ref. 47).

to train subjects on several pairs (e.g., three pairs ${ }^{63}$, four pairs ${ }^{16}$ or eight pairs $2,16,23$ ) of stimuli concurrently, combining trials of each pair within each session; the basic procedure for concurrent discrimination learning is identical to that provided below. In some cases, pairwise visual discrimination may serve as the first stage in a more complex task, such as transverse patterning ${ }^{34}$. To test retention, another possible post-training manipulation is to retest animals after a delay (e.g., 5-7 d) with the same set of stimuli with which they were trained. Retention tests can be used to assess the effects of pharmacological or other manipulations on previously acquired visual discriminations ${ }^{14}$ or to test hypotheses about the nature of acquisition learning ${ }^{6}$. Note that it is possible to test the same group of animals on more than one discrimination, e.g., to test mice with 'marble-fan' stimuli followed by photographic stimuli, although transfer effects are possible (see the discussion of the flexible battery approach above).

Typically, visual discrimination acquisition performance is assessed in terms of percentage accuracy in the form of an acquisition curve and/or in terms of the number of sessions, trials and errors (incorrect responses to noncorrection trials) required to reach criterion. In addition, latencies, percentages of bias and perseveration scores may be analyzed. We refer the reader to the general considerations above and to the PROCEDURE for further details.

Object-location paired-associates learning (Step 10B). An object-location paired-associates learning session (Fig. 4) begins with a free reward delivery and illumination of the magazine light, indicating that a trial may be initiated (as in pretraining). After initiation, two stimuli are presented, composing one of six trial types (Fig. 5a). A response can be made to the $S^{+}$(object in the correct location, i.e., a correct response) or the $S^{-}$(object in the incorrect location, i.e., an incorrect response). After a correct response, the stimuli are removed from the screen and a reward is delivered in conjunction with a tone $(1 \mathrm{~s}, 3 \mathrm{kHz})$ and magazine illumination. When the animal enters the magazine to retrieve the reward, the magazine light is turned off and an ITI begins, after which the magazine is again illuminated to indicate that a new trial may be initiated. After an incorrect response, the stimuli are removed from the screen and the house light is inverted for a 5-s time-out period, after which the ITI begins. After the ITI, the magazine is illuminated for trial initiation, but the next trial will be a CT (see General considerations above). Excluding CTs, there are an equal number of presentations of each trial type in each session, in a pseudorandom sequence (maximum of three consecutive presentations).

This protocol for rats and mice is based on that first described by Talpos et al. ${ }^{41}$, and it uses the 'flower-plane-spider' stimulus combination described in published work (Fig. 5a). However, we note that recent rat task development has led us to use line patterns as objects instead (Fig. 5b), on the basis of preliminary data indicating reduced variability when using patterns compared with images. In addition, although we present the task here with no consequences for touches to the blank location when stimuli are presented on the screen (as in previous publications), we are currently using a method in which we follow blank touches by stimulus offset and a CT (Fig. 4).

To test whether animals form specific object-location associations during the task-as opposed to acquiring a set of trial type-specific conditional responses-one can run a probe test in which trials consist of the presentation of two copies of the same object, one in that object's correct location and the other in one of that object's two incorrect locations (e.g., $\mathrm{S}^{+}=$object 1 in location $1, \mathrm{~S}^{-}=$object 1 in location 2 , referred to as sPAL (samePAL) in ref. 41). Although Talpos et al. ${ }^{41}$ assessed the difference between the standard object-location paired-associates learning task training and the same-object probe by using a between-subject design, the common approach since developed involves running two sessions of the same object probe after stable performance on the standard task has been established. The degree to which an 


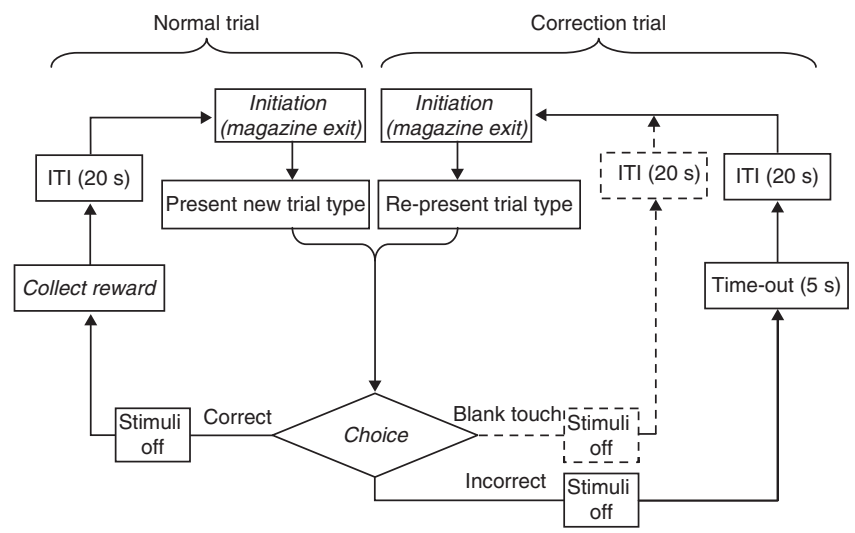

Figure 4 | Flowchart overview of object-location paired-associates learning task. After initiation, one of six possible trial types (Fig. 5), each composed of one $\mathrm{CS}^{+}$and one $\mathrm{CS}^{-}$, is presented on the screen. Correct responses (to $\mathrm{CS}^{+}$) are rewarded, and after reward collection and an ITI a new trial may be initiated. Incorrect responses (to $\mathrm{CS}^{-}$) are discouraged with a time-out, and then after an ITI and initiation the previous trial type is represented (a CT). The CT loop will continue until a correct response is made. In our recent task development, we have introduced a consequence for touching the blank location (dashed lines). The labels in italics indicate steps in which the animal is required to perform an action.

animal's performance drops during this probe test is interpreted as reflecting the extent to which the animal was solving the original task according to alternative, nonconfigural strategies.

Typically, object-location paired-associates learning acquisition performance is assessed in terms of percentage accuracy in the form of an acquisition curve, and/or in terms of the number of sessions or trials required to reach criterion (see ANTICIPATED RESULTS). In addition, errors, latencies, percentages of bias and perseveration scores may be analyzed. In addition to these performance measures, preliminary evidence from our laboratory indicates that all trial types are not always acquired at an equal rate, particularly 'flower-plane-spider' stimuli when acquired by rats (Fig. 5a). Therefore, separate trial-type performance analysis may be performed, as performance differences may be more pronounced depending on the trial type. We refer the reader to the general considerations above and to the PROCEDURE for further details.

VMCL (Step 10C). The protocol described here is the most recent for rats; the task is still in development for the mouse. Building on previous publications ${ }^{2,23,74,97}$, the present protocol includes an additional phase of VMCL-specific pretraining after standard pretraining and immediately before VMCL training. This phase addresses several potential concerns. First, it counteracts any initial side bias that subjects may have by requiring responses to both flanking locations. Second, it accustoms the subject to making two responses for a reward, which is in contrast to the single response required for reward during pretraining. Third, it provides an opportunity to introduce a limited hold ( $\mathrm{LH})$ period.

Each VMCL-specific pretraining session begins with a free reward delivery and illumination of the magazine light, indicating that a trial may be initiated (as in pretraining). After initiation, a plain white square is presented in the central location, which remains on the screen until it is touched (touches to the two blank locations are ignored). When the central stimulus is touched by the subject, it disappears and is replaced by another stimulus (also a plain white square) in one of the two flanking locations (left and right; 1 and 3). Excluding CTs, the same location is not used more than three times consecutively, and each location is used in five out of every ten trials. This second stimulus remains on the screen for the LH period (usually $2 \mathrm{~s}$ ), or until a response is made. Touches to the central location are ignored. After a correct response (stimulus touched within the LH period), the stimulus disappears, a reward and tone $(1 \mathrm{~s}, 3 \mathrm{kHz})$ are delivered and the magazine is illuminated. When the animal enters the magazine to retrieve the reward, the magazine light is turned off and an ITI begins, after which the magazine is again illuminated to indicate that a new trial may be initiated. Following an incorrect response (blank peripheral location touched within the LH period), the stimulus disappears, the house light is inverted for a 5-s timeout period and then the ITI begins. After the ITI, the magazine is illuminated and a CT may be initiated (see General considerations above). If the subject fails to respond during the LH period, the consequences are the same as those for an incorrect response. The purpose of the LH is to ensure that the subject responds to the flanking stimulus while it is still at the screen after making the initial response to the central stimulus (e.g., the rat makes a head turn while rearing).

VMCL task trials progress in a similar manner (Fig. 6). However, instead of a plain white square stimulus in the central location, one of two discriminative stimuli is presented (Fig. 7). Excluding CTs, the same stimulus is not used more than three times consecutively, and each stimulus is used in five out of every ten trials. When the subject touches the discriminative stimulus, it remains on the screen and two choice stimuli are also presented: a plain white square in each of the two flanking locations (left and right). These remain on the screen until one is touched, or until

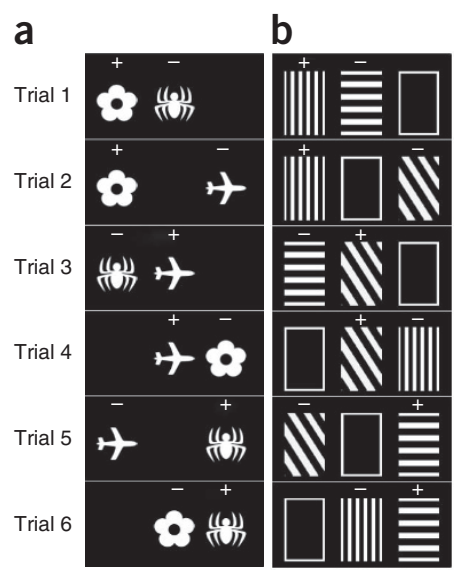

Figure 5 | The six possible trial types in the object-location pairedassociates learning task. $(\mathbf{a}, \mathbf{b})$ The stimuli in the left panel $(\mathbf{a})$ are the basis of this protocol and of all published material using the touchscreen object-location paired-associates learning task. However, in our recent rat task development, we have used the stimuli in the right panel (b). $\mathrm{CS}^{+}$(correct choice) is denoted ' + '. CS- (incorrect choice) is denoted ' - '. When standard stimuli are used (a), touches to the blank location are ignored. When stimuli in $\mathbf{b}$ are used, the blank location is framed white, and touches to it are discouraged (Fig. 4). Panel $\mathbf{a}$ is reproduced with kind permission from ref. 41 . 


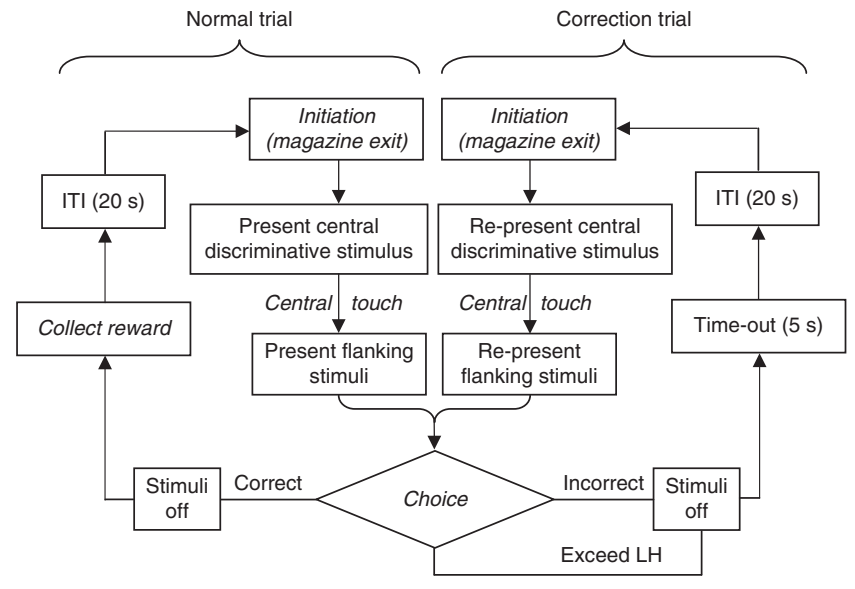

Figure 6 | Flowchart overview of the VMCL task. After initiation, one of two discriminative stimuli is presented. Touching this stimulus results in the additional presentation of two choice stimuli in the flanking locations (left and right). The correct/incorrect response choice is determined by the discriminative stimulus, e.g., stimulus $\mathrm{A}$ indicates that right is correct. The subject must respond within the LH period (usually $2 \mathrm{~s}$ ). Correct responses are rewarded, and after reward collection and an ITI a new trial may be initiated. Incorrect and absent (LH exceeded) responses are discouraged with a time-out, and then after an ITI and initiation the previous trial is re-presented (a CT). The CT loop will continue until a correct response is made. VMCL-specific pretraining trials progress in a similar manner, with some differences. The central stimulus is plain white, rather than discriminative. When it is touched, it is removed and replaced with a single plain white flanking stimulus. Finally, an 'incorrect' response is instead defined as touching the blank flanking location. The labels in italics indicate steps in which the animal is required to perform an action.

the LH (2 s) is exceeded. Touches to the discriminative stimulus are ignored. The nature of the stimuli is counterbalanced, but, for example, if stimulus A is presented, then the left stimulus is correct and the other is incorrect, whereas if stimulus B is presented the right stimulus is correct. Again, three response types are possible; the definitions and consequences of these are as in VMCL-specific pretraining, except that an incorrect response is now defined as a response to the incorrect stimulus (rather than to the blank peripheral location).

Depending on the aims and hypotheses of the researcher, the reward contingency may be reversed after acquisition in order to test reversal learning (and thereby assess cognitive flexibility) $)^{74}$.

Typically, VMCL performance is assessed in terms of percentage accuracy in the form of an acquisition curve (if all subjects complete a certain minimum number of sessions, e.g., 5, 10 $)^{74,97}$, and/or in terms of the number of sessions, trials and errors required to reach criterion $23,74,97$. In addition, average correct and incorrect response latency, average magazine latency 35,83 , percentage of bias $^{23,74}$ and perseveration score ${ }^{74}$ may be analyzed. Errors to acquisition criterion may be split into those committed in three distinct phases of learning-chance, early and late-which (in a session comprising 100 trials) may be defined as performance levels of $\leq 61 \%, 61-70 \%$ and $71-85 \%$, respectively ${ }^{23}$. Errors to reversal criterion may also be split into those committed in distinct phases of learning, e.g., into prechance and above, which (in a session comprising 100 trials) may be defined as performance levels of $\leq 38 \%$ and $39-85 \%$, respectively ${ }^{74}$. The number of sessions required to complete
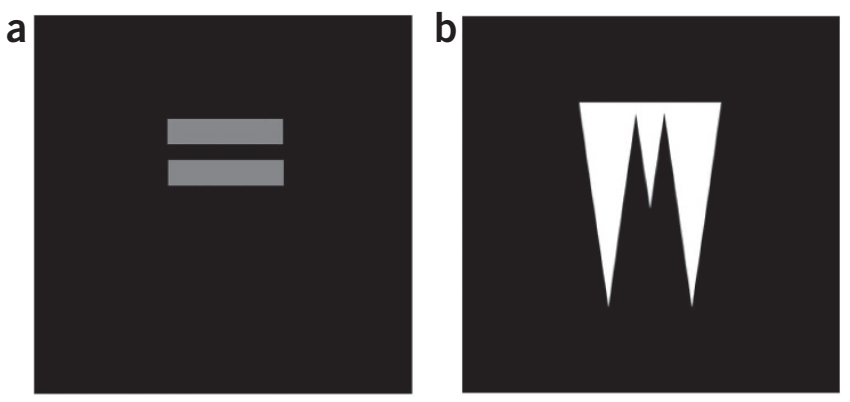

Figure 7 | VMCL discriminative stimuli. (a,b) Reward contingencies are counterbalanced but, for example, stimulus A (a) may indicate that the right choice stimulus will be correct (and left incorrect), whereas stimulus B (b) indicates that left will be correct (and right incorrect). Figures courtesy of Campden Instruments.

the VMCL-specific pretraining phase may also be calculated. We refer the reader to the General considerations section above and to the PROCEDURE for further details.

Autoshaping (Step 10D). Autoshaping in rats and mice may be conducted using Campden touchscreen chambers, which are suitably equipped (see MATERIALS) ${ }^{8}$. Previous work (in rats, but not mice) has also used bespoke apparatus built in-house at the University of Cambridge. During a trial, a white rectangular stimulus is presented on one side of the screen (the left or right) for a prespecified stimulus duration (standard: $10 \mathrm{~s}$ ) (Fig. 8). A stimulus on one side of the screen (e.g., left) is designated as the $\mathrm{CS}^{+}$and the other as the $\mathrm{CS}^{-}$, counterbalanced across subjects. Upon $\mathrm{CS}^{+}$offset, a tone $(1 \mathrm{~s}, 3 \mathrm{kHz})$ is emitted, a reward is delivered to the magazine and the magazine is illuminated. Upon $\mathrm{CS}^{-}$offset, there is no tone or reward. Infrared (IR) photobeams in front of each side of the screen detect approaches to each side, and entries to the reward collection magazine are also detected. Following stimulus offset (and, if reward was delivered, entry into

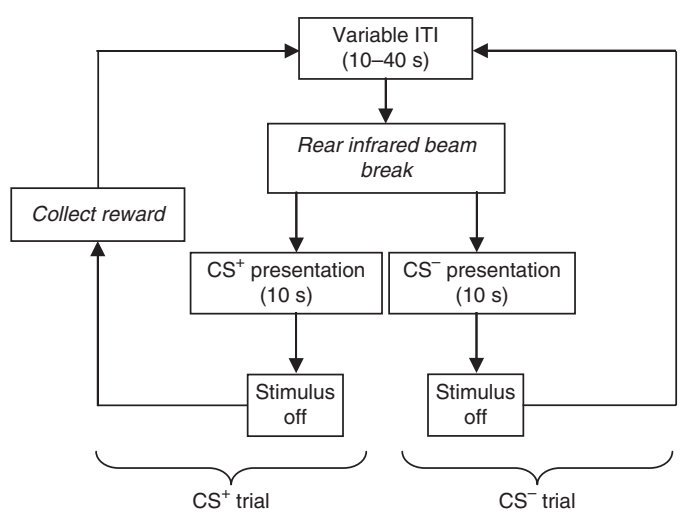

Figure 8 | Flowchart overview of the autoshaping task. After a variable ITI, a trial is initiated when the animal breaks the IR beam at the rear of the chamber and a stimulus is displayed (CS+ or $\left.^{-} \mathrm{CS}^{-}\right)$. Regardless of the animal's behavior, stimulus offset occurs after a prespecified display time. Upon $\mathrm{CS}^{+}$offset, a reward is delivered, and when the animal enters the magazine to collect it another variable ITI begins. Upon CS- offset, reward is not delivered and another variable ITI begins. CS+ and CS- trials are organized in pairs, such that if $\mathrm{CS}^{+}$is presented first a CS- trial follows. The labels in italics indicate steps in which the animal is required to perform an action. 
the magazine for reward collection), a variable ITI (standard range: 10-40 s) begins, after which the animal must break the IR photobeam near the rear of the chamber (opposite the touchscreen) to initiate the next trial. Initiation is followed immediately by a click $(0.2 \mathrm{~s})$ and stimulus onset. This maximizes the probability that the animal will be able to view both sides of the screen upon stimulus presentation and also minimizes inadvertent stimulus approaches. The house light is off throughout the task. Each pair of trials comprises one $\mathrm{CS}^{+}$trial and one $\mathrm{CS}^{-}$trial, such that each 40-trial session includes 20 presentations of each type.

Depending on the autoshaping results obtained and hypotheses being tested, an 'omission' probe phase may be performed to assess the nature of the associations governing responding. Sessions in this probe phase are identical to those in autoshaping, except that approach to the $\mathrm{CS}^{+}$prevents reward delivery. If the previously acquired autoshaping response is governed by a Pavlovian association, stimulus discrimination (as measured by approaches) should be resistant to reward omission across multiple sessions.

Autoshaping is preceded by stage 1 of standard pretraining, and by a unique pretraining phase in which reward is delivered after a variable ITI ( $0-30 \mathrm{~s}$; additional time allowed if necessary to ensure animal is not in the magazine when ITI ends), with the magazine illuminated and a tone emitted upon delivery. The animal must enter the magazine to collect the reward (upon which the magazine light is extinguished) in order to initiate the next delay period.

The primary performance measures in this task are the number and latency of approaches to the $\mathrm{CS}^{+}$and $\mathrm{CS}^{-}$side of the chamber. The number and latency of touches to the $\mathrm{CS}^{+}$and $\mathrm{CS}^{-}$side of the screen are also recorded. Following initial chamber habituation and training, this task is acquired rapidly, with both control rats and mice displaying clear $\mathrm{CS}^{+} / \mathrm{CS}^{-}$discrimination within five daily sessions.

\section{MATERIALS}

\section{REAGENTS}

- Rats or mice (see Reagent Setup)

- Animal housing (see Reagent Setup)

- Rodent food pellets (e.g., rodent pellets, Special Diets Services)

- Rewards: we use solid (e.g., Bio-Serv purified rodent dustless precision pellets, $45 \mathrm{mg}$ (rat)/14 mg (mouse), through Sandown Scientific) or liquid (Yazoo strawberry milkshake, FrieslandCampina UK) food rewards I CAUTION When you fill the reward dispenser with dustless precision pellets, take care to discard any dust, as this can potentially clog dispensers. I CAUTION All liquid reward containers and delivery lines should be thoroughly rinsed at the end of each testing day to prevent clogging and/or the growth of potentially harmful microorganisms.

- Cleaning materials (e.g., TriGene, $70 \%$ (vol/vol) ethanol solution, stiff brush)

\section{EQUIPMENT}

- Sound- and light-attenuating box with ventilation system, enclosing an operant chamber and reward delivery system

- Touchscreen operant chambers (from, e.g., Campden Instruments, Med Associates, other commercial suppliers; or a custom-made operant system). Note that these are species-specific. Rodent touchscreen operant chambers made by different companies may vary, but share many common features. The specific model used depends on the experimenter's needs and preference. In Equipment Setup we describe mouse and rat chambers from Campden Instruments and our in-house assembled boxes

- Camera above the chamber, connected to a closed-circuit monitor and digital video recording device, to monitor and record animals' behavior (optional but recommended)
- Controlling software and devices (generally available from the operant chamber supplier)

- Black plastic masks with response windows (the number and size of which differ between tasks; see Table 1 and Fig. 9)

- Shelf for rat chamber (for some tasks, see Equipment Setup)

- Appropriate data analysis software

- Personal protection equipment (e.g., disposable medical gloves, lab coat or coverall, FFP2 mask) should always be worn when you are handling or working near animals, in order to minimize allergen exposure

\section{REAGENT SETUP}

Rodents Laboratory-bred or commercially available rats/mice are generally used for testing. There are some advantages to testing male rodents, such as avoiding potential estrus cycle-related performance variability in females ${ }^{119,120}$, and potentially increased inter-male aggression when males must be tested in the same apparatus as females. Most commonly, we use Lister hooded rats and mice on the C57BL/6 or 129 substrain genetic backgrounds, and we prefer beginning training when rodents are young adults, e.g., 10- to 14 -week-old mice. However, females ${ }^{51,55,57,58,60}$, aged rodents ${ }^{12}$ and various strains ${ }^{16,49,52,121}$ have been tested. Choice of animals is an important consideration for all cognitive and behavioral testing of rodents. ! CAUTION All experiments using live animals must be approved by national and institutional bodies, and performed according to their regulations.

! CAUTION If animals are not fully grown when food restriction begins, they must be allowed to gain sufficient weight as they continue to grow. Standard strain growth curves are available for guidance (e.g., http://jaxmice.jax. org/support/weight/index.html). a

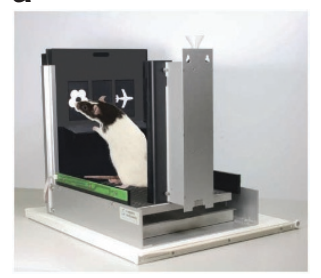

b

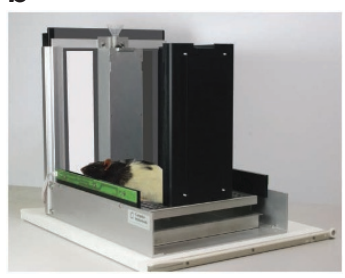

C

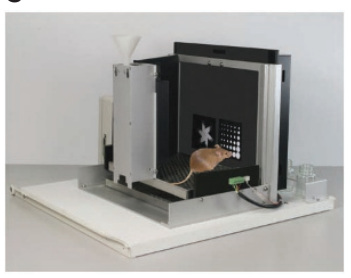

d

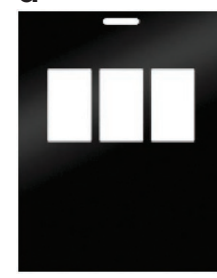

e

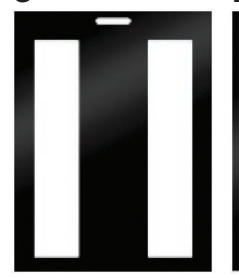

f

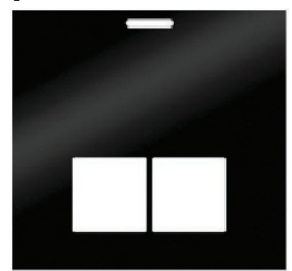

Figure 9 | Illustrations of Campden Instruments touchscreen chamber apparatus. (a) Normal rat chamber configuration, with shelf, showing a rat performing object-location paired-associates learning. (b) Autoshaping rat chamber configuration, showing a rat performing the autoshaping task. (c) Normal mouse chamber configuration, showing a mouse performing visual discrimination. (d-f) Black plastic masks, which are used to cover the touchscreen in a-c. Images courtesy of Campden Instruments. 
Animal housing Rats and mice usually should be housed in groups (e.g., 2-5), with sawdust, bedding and (optional, although recommended) shelter (or alternatives). Cages, bedding and so on should be changed or cleaned weekly. The housing room should be maintained at a constant temperature $\left(21 \pm 2{ }^{\circ} \mathrm{C}\right)$ and humidity $(55 \pm 10 \%)$. Lighting is usually on a 12-h light-dark cycle, with lights off at 7:00 a.m. or 7:00 p.m. We favor lights off at 7:00 a.m., so that rodents can be tested in the active period of their circadian cycle. To our knowledge, conducting behavioral testing during the dark phase of an inverse light cycle has no adverse effect on the welfare of mice, but may improve activity levels, learning and memory ${ }^{122-124}$. However, researchers should be aware that lighting phase could potentially interact with sex, strain, experimental manipulations and so on to influence performance. When shifting or inverting the light cycle of rodents, allow sufficient time for rodents to become fully entrained to the inverse cycle before commencing behavioral testing ${ }^{125}$. We tend to allow $1 \mathrm{~d}$ per hour of shift. This, of course, is an important consideration for all cognitive and behavioral testing of rodents.

\section{EQUIPMENT SETUP}

Campden operant chambers Housed inside a dense fiberboard box, these are equipped with a fan (for ventilation and masking extraneous noise), touchscreen monitor (rat: 15.0 inch, screen resolution 1,024 $\times 768$ (rotated); mouse: 12.1 inch, screen resolution $600 \times 800)$, tone and click generator, house light (light-emitting diode), magazine unit (with light and IR beam to detect entries; in the standard configuration, this is outside the testing arena, on the wall opposite the touchscreen) and a pellet dispenser and/or pump connected to bottles of liquid reward (see Fig. 10 for the rat chamber). The chambers have a trapezoidal shape (in $\mathrm{cm}$, rat: 30 high $\times 33$ long (screen-magazine) $\times 25$ wide (at screen) or 13 wide (at magazine); mouse 20 high $\times 18$ long $\times 24$ or 6 wide), which is composed of three black plastic walls opening onto the touchscreen. This shape is intended to help focus the animal's attention to the touchscreen and the reward delivery area. The touchscreen uses IR photocells, and therefore does not require the subject to exert any pressure for responses to be registered. Our experience is that rodents work most readily and learn fastest with these IR beams, and not when they have to exert any pressure on the screen, although we have not carried out a properly controlled experiment to test this idea. We typically observe rodents responding to the screen with their noses (Supplementary Video 1). Access to the chamber is through a transparent lid, which can be secured to the trapezoidal walls with latches during animal testing. The floor consists of perforated stainless steel, raised above a tray lined with filter paper. Two additional photobeams extend between the side walls of the arena, parallel to the screen, to detect the movement of an animal in the front (rat: $\sim 6 \mathrm{~cm}$ from the screen; mouse: $\sim 7 \mathrm{~cm}$ ) or the rear (rat: $\sim 5 \mathrm{~cm}$ from the magazine; mouse: $\sim 3.5 \mathrm{~cm}$ ) parts of the arena. A small IR camera can be installed above the chamber to monitor animals' behavior (optional but recommended). In rat chambers, attaching a shelf to the mask has proved to be effective at reducing impulsive responses and improving attention directed to the stimuli, by forcing the rat to rear up before making a choice ${ }^{2}$. In Campden rat chambers, a spring-hinged shelf $(24 \mathrm{~cm}$ wide $\times 6 \mathrm{~cm}$ long $)$ can be attached $15 \mathrm{~cm}$ above the floor at a $90^{\circ}$ angle to the screen and mask. Our laboratory uses these shelves for rats in the majority of tasks (the exception herein being autoshaping). Campden Instruments provides advice on setting up the touchscreen equipment, including touchscreen and reward dispenser calibration.

Our in-house operant chambers Housed inside a melamine box, chambers (modified in our lab from Med Associates operant chambers) are equipped with a fan, IR touchscreen monitor (in cm, rat: 29.0 high $\times$ 23.0 wide; mouse: 16.0 high $\times 21.2$ wide; Craft Data Limited), tone generator, click generator, house light $(\sim 3 \mathrm{~W})$, magazine and pellet dispenser. The touchscreen does not require the subject to exert any

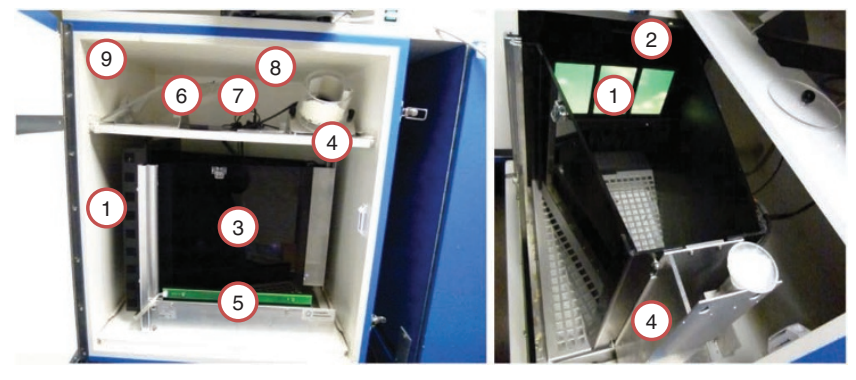

Figure 10 | Annotated photographs of a Campden Instruments rat touchscreen chamber. (1) Touchscreen, (2) black plastic mask covering the touchscreen except for response windows, (3) black Perspex walls, (4) pellet dispenser (optional), (5) IR beam assembly, (6) house light positioned above the chamber, (7) IR camera positioned above the chamber, (8) tone and click generator and (9) sound/light-attenuating box with a ventilation fan fitted.

pressure in order for touches to be registered. The chambers have a rectangular shape, consisting of a metal frame with clear Perspex walls (in $\mathrm{cm}$, rat: 29 high $\times 31$ long $\times 24$ wide; mouse: 13 high $\times 25$ long $\times 19$ wide ; excluding space below floor). Access is through a hinged side wall, secured with a latch during testing. The floor consists of stainless steel bars spaced $1 \mathrm{~cm}$ apart above a tray lined with filter paper. The magazine is equipped with a light and a photocell nose-poke detector. A spring-hinged shelf $(20.5 \mathrm{~cm}$ wide $\times$ $6 \mathrm{~cm}$ long) is also fitted in rat chambers $14.0 \mathrm{~cm}$ above the floor, at a $90^{\circ}$ angle to the screen and mask.

Masks and stimuli A black plastic mask (in cm, rat in-house: 38.7 high $\times 30.0$ wide; rat Campden: 35.8 high $\times 28.0$ wide; mouse in-house: 11.8 high $\times$ 22.8 wide; mouse Campden: 24.3 high $\times 28.0$ wide) with response windows is fitted in front of the touchscreen to reduce accidental screen touches and make response locations clearly identifiable from the background. These have varying numbers and sizes of response windows, depending on the task (Table 1). Autoshaping As far as we know, this task can only be run in the Campden chambers described above at the present time. In contrast to the usual chamber configuration, the reward collection magazine unit is positioned immediately in front of the center of the touchscreen, inside the arena (Fig. 9b). The photobeam that usually traverses the width of the chamber in front of the screen is split into two independent beams by the magazine, such that approaches to each side of the screen can be measured separately. An additional photobeam traverses the side of the box opposite the screen as in the normal setup. ! CAUTION When the apparatus is used in the 'autoshaping configuration', a fitted cover must be used to seal the hole in the chamber walls that usually allows access to the externally located magazine. Ensure that the magazine is secured correctly to prevent possible injury to the subject in the arena. In addition, ensure that the IR beam microswitch is set to the autoshaping configuration (as explained in the manufacturer's manual). Controlling software and devices Controlling software can be purchased from the suppliers of the operant chambers, e.g., Whisker ${ }^{126}$ and ELO software (ELO Touchsystems). Multiple chambers may be controlled by a single computer, although it is important to check that minimum system requirements are met (e.g., memory and graphics cards) to prevent delays in stimuli presentation and chamber responses. All task software is designed on the basis of earlier publications and is available (excluding, in some cases, recent modifications) from Campden Instruments, Med Associates (K-Limbic) or other suppliers. Alternatively, software may be programmed in common programming languages, such as Visual Basic 6.0 (Microsoft). 


\section{PROCEDURE}

\section{Preparation for pretraining}

1| If it is not necessary to transport animals to the facility from an external source, proceed directly to Step 2 of the protocol. If transportation is necessary, do so and follow it by an acclimatization period of $7 \mathrm{~d}$ (minimum). During these $7 \mathrm{~d}$, provide animals with ad libitum food and water and conduct no procedures. You may begin handling and weighing the animals after $2 \mathrm{~d}$ of acclimatization. Proceed to Step 3 after the 7-d acclimatization period.

$\triangle$ CRITICAL STEP When you plan and design experiments, we advise that you consult with your institutional animal care regulatory body regarding matters such as food restriction and housing.

$\Delta$ CRITICAL STEP Some cohorts of mice have relatively high between-subject variability, and thus larger $n$ numbers are required. There are many variables that can affect variability, such as strain, maternal care, events during transportation and so on. We advise minimizing the age range of cohorts to reduce potential age-related variability. When possible, calculation of $n$ numbers should be done in accordance with a power calculation that is based on previous work with that strain of animal, ideally from the same supplier. This, of course, is an important consideration for all cognitive and behavioral testing of rodents.

$\triangle$ CRITICAL STEP Train all animals by using this preparation and pretraining process (Steps 1-9) before their first instrumental touchscreen task (Steps 10A, B or C). If subjects have previously been trained and tested on another instrumental touchscreen task in the battery, maintain food restriction and start at pretraining Step 9. For pretraining before autoshaping (Step 10D), proceed to Step 10D after Steps 1-5. As discussed in Experimental design, touchscreen tasks (e.g., Steps 10A-C, also see refs. 4,5) may be used in flexible combinations and orders.

2| Weigh each animal for 3 consecutive days with ad libitum food and water, and then calculate the mean free-feeding weight of each animal.

$\triangle$ CRITICAL STEP Ensure that each animal can be reliably identified.

3| Begin food restriction. Adhere to all relevant institutional and governmental animal handling guidelines. Slowly reduce (e.g., over 3-7 d) the weight of individual animals down to the goal weight, which will be a percentage of the measured free-feeding weight (e.g., we use $85-95 \%$, which is in line with our institutional guidelines) by controlling the daily amount of food they are given (e.g., for rats, $\sim 7 \mathrm{~g}$ of food per $100 \mathrm{~g}$ of body weight; for mice, $\sim 2-3 \mathrm{~g}$ of food per $25-35 \mathrm{~g}$ of mouse). Start Step 4 when animals are close to their goal weights. Maintain food restriction throughout touchscreen testing. $\triangle$ CRITICAL STEP It is important to check the weight of animals daily (mice) or twice a week (rats) until the target weight is reached. This also helps habituate the animals to being handled. Aim to avoid weight reduction of greater than $5 \%$ per day, and weight reduction below $85 \%$ of free feeding.

4| Introduce reward (pellets or milkshake) inside the cage to habituate the animals for 1-3 d. Solid rewards may be scattered on the cage floor; liquid rewards should be put into a shallow, wide-based dish.

\section{Pretraining}

5| Set up the apparatus (see MATERIALS) for this pretraining stage (stage 1), with all electronic components on so that subjects may habituate to these. Here and in all subsequent steps, use touchscreen masks and stimuli as appropriate for the task (e.g., visual discrimination; see Experimental design, MATERIALS and Table 1). Note that for the VMCL task, only locations 1 (leftmost) and 3 (rightmost) should be used during pretraining Steps 7-9. It is not necessary to run any software during stage 1, but we recommend recording subjects' activity if the necessary apparatus and software are available (e.g., chambers from Campden Instruments). Place 10 reward pellets or $0.2 \mathrm{ml}$ of liquid reward in the magazine of each chamber (if the computer program you are using does not do this automatically). Place each rodent in its assigned chamber for 30 min. Remove the rodent and check that the reward has been consumed. Return each animal to its respective home cage. Test all subjects on stage 1 for at least two sessions. The criterion for advancing to the next step is consuming all rewards in a session.

$\triangle$ CRITICAL STEP Animals require fewer standard rodent food pellets when receiving rewards during training; adjust daily food allowance as appropriate to maintain goal weight.

$\Delta$ CRITICAL STEP Aim to train, weigh and feed each animal at approximately the same time each day, and use the same operant box for each animal during training. Always counterbalance chambers and testing times across experimental groups. It is good practice to weigh mice daily, but once or twice per week may be sufficient for rats. We recommend one session per day, 5-7 d per week.

$\triangle$ CRITICAL STEP Advance individual subjects to the next pretraining stage when they reach criterion, even if some animals in the group remain in the previous stage(s).

$\triangle$ CRITICAL STEP To ensure that the touchscreen and IR photobeams retain maximum sensitivity and to prevent accumulation of dirt and excrement, operant chambers should be cleaned regularly (e.g., once a week or more). We typically dismantle the inner chambers (as much as possible) and clean them with surface disinfectants (e.g., TriGene and 70\% (vol/vol) ethanol) and paper towels or a stiff brush. 
6| Set up the apparatus as detailed in MATERIALS and the software program for this stage (stage 2) with settings as detailed in Experimental design. Place each subject in its assigned chamber and start the session. The session finishes after 60 min or after 100 trials (rat) or 30 trials (mouse) are completed (whichever comes first). (For pretraining before mouse object-location paired-associates learning, there are 36 trials per session.) After session termination, return each animal to its respective home cage. Advance individual subjects to the next training phase when they achieve a criterion of completing all trials (mice) or 60 trials (rats) within 60 min.

$\triangle$ CRITICAL STEP At the end of each session, record the most crucial data for each subject (e.g., number of correct responses, number of trials completed) in case of computer malfunction. However, most software programs will record many other measures (see Experimental design).

$\triangle$ CRITICAL STEP If you are testing the effects of a manipulation conducted before onset of the experiment (case 1, see Experimental design), ensure that animals in experimental and control groups complete comparable numbers of trials per session. Cap the number of trials given per session to accommodate the lowest responders.

7| Repeat Step 6 (stage 2) for stage 3, using the appropriate software program (see Experimental design).

8| Provide a single free reward (if your program does not do this automatically) for stage 4. 0therwise, proceed as in Step 6 (stage 2), but use the appropriate software program (see Experimental design).

9| Proceed as in Step 8 (stage 4) for stage 5, using the appropriate software program (see Experimental design). The criterion for completing this stage is completing all trials with $\geq 80 \%$ correct (not including CTs) within 60 min (rat), or with $\geq 75 \%$ correct within $35 \mathrm{~min}$ (mouse), on two consecutive sessions. (Allow $40 \mathrm{~min}$ for mice in pretraining for object-location paired-associates learning, in which mice receive 36 trials per session.)

$\triangle$ CRITICAL STEP There is likely to be variation in the number of days that animals require to complete pretraining. We suggest resting animals when they reach criterion (with reminder sessions), and then rebaselining all subjects so that the entire group can advance to a specific touchscreen task on the same day (see Experimental design). If subjects are scheduled to receive experimental treatments after pretraining but before task acquisition (case 2, see Experimental design), perform these now (after Step 9), making sure to counterbalance control and experimental groups according to the number of sessions required to complete pretraining. Rebaseline the subjects on Step 9 (stage 5) before task-specific training.

\section{Task}

10| Proceed to visual discrimination (option A), object-location paired-associates learning (option B), VMCL (option C; rats only) or autoshaping (option D).

\section{(A) Visual discrimination}

(i) Visual discrimination acquisition training. When subjects are ready for task training to begin, counterbalance stimulus reward contingencies (such that approximately half of each group receives stimulus $\mathrm{A}^{\text {as }} \mathrm{CS}^{+}$and $\mathrm{B}$ as $\mathrm{CS}^{-}$, and the rest the reverse), according to the number of sessions required to complete pretraining.

(ii) Begin training on once-daily sessions of visual discrimination acquisition, 5-7 d per week. Provide a single free reward (if your program does not do this automatically). Set up the apparatus as detailed for this task in MATERIALS and the software program for this stage with settings as detailed in Experimental design, with reward contingencies as appropriate for each subject. Place each subject in its assigned chamber, and start the session. The session finishes either after $60 \mathrm{~min}$ or after 100 trials (rat) or 30 trials (mouse) are completed (whichever comes first). After session termination, return each animal to its respective home cage.

$\triangle$ CRITICAL STEP Give careful consideration to the stimulus set you choose (Fig. 3a-d). Standard rat stimuli ('spider' and 'plane') are also standard stimuli used in the object-location paired-associates learning task, and thus they should be avoided here if rats have previously been or may subsequently be tested on object-location paired-associates learning. If you wish to use morphed stimuli as a post-training manipulation, this may also affect your initial choice of stimuli. $\Delta$ CRITICAL STEP Carefully monitor visual stimulus biases on the first day of testing (see Step 10A(v)). If animals show strong stimulus biases, consider revising the stimuli. This, of course, is an important consideration for all cognitive and behavioral testing of rodents involving object discriminations.

$\triangle$ CRITICAL STEP Given that performance is likely to be poor at the start of training, with animals thus receiving many CTs per trial, limit sessions to 50 trials (rat) or 15 trials (mouse) in $60 \mathrm{~min}$, for at least two sessions. Continue until the subjects can complete this in $30 \mathrm{~min}$. Give each subject an even number of these reduced sessions, such that they can be combined into full 100- or 30-trial sessions for analysis. If the subject completes fewer trials than required, the missed trials may be added to the trials required in the next session (if fewer than 10 ) or given in a new session. $\Delta$ CRITICAL STEP If you are testing the effects of a manipulation conducted before onset of task acquisition (e.g., cases 1 and 2, see Experimental design), ensure that the animals in experimental and control groups complete 
comparable numbers of trials per session throughout task acquisition. Cap the number of trials given per session to accommodate the lowest responders.

$\Delta$ CRITICAL STEP At the end of each session, record the most crucial data for each subject (e.g., number of correct responses, number of trials completed) in case of computer malfunction. However, most software programs will record many other measures (see Experimental design).

(iii) Continue training on once-daily sessions of visual discrimination acquisition, 5-7 d per week, until the animals have reached the acquisition criterion for this task, at which point you may proceed to the next step. The acquisition criterion for this task is the completion of all trials with an accuracy of $80 \%$ or, alternatively, $85 \%$ (refs. $13,51,58$ ) (excluding CTs) for two consecutive sessions. If you are testing animals that received experimental manipulations before task acquisition (cases 1 and 2, see Experimental design), the main experimental readouts may be differences in the rates of acquisition (and/or final performance levels). If so, continue training all animals on all sessions, either for a given number of sessions (to allow plotting of an acquisition curve) or until the control and/or experimental group(s) attain criterion or stable performance. Alternatively, if post-training behavioral challenges are to follow (e.g., morphed visual stimuli, retention and reversal; see Step 10A(iv)), and/or when postacquisition manipulations are to be conducted (e.g., cases 3 and 4 in combination with continued training at asymptotic performance level, see Experimental design), we suggest resting animals, with reminder sessions, when they reach criterion, until the entire group has achieved criterion, at which time the entire group may be rebaselined before progressing to Step 10A(iv) (see Experimental design for details and alternatives). If subjects are scheduled to receive experimental treatments after acquisition but before Step 10A(iv) (case 3, see Experimental design), perform these when all animals have reached criterion (at least) once, being sure to counterbalance control and experimental groups according to acquisition performance. Rebaseline the subjects before proceeding.

$\Delta$ CRITICAL STEP To investigate the effects of postacquisition treatment (cases 3 or 4 ) on behavioral challenges using stimuli that are not part of regular task acquisition (e.g., morphed stimuli, Step 10A(iv)), animals should also be briefly (e.g., for one or two sessions) exposed to these before treatment to avoid confounds due to novelty or contextual change, and to allow for a within-subject pre- and post-treatment comparison of performance level. In the case of microinfusion studies, animals should be rebaselined after surgery until a stable performance level is reached, and they should be introduced to the relevant novel stimuli at this point. Before commencing subsequent vehicle and drug infusion, a mock infusion involving the insertion of the infusion cannula only should be performed; this should be followed by a vehicle infusion to assess nonspecific effects on performance.

(iv) Post-training experimental manipulations. Depending on the aims of the experiment, proceed with appropriate post-training manipulations. Various post-training manipulations are possible. For continued training at the asymptotic performance level, conduct the experiment as in Step 10A(ii). Transient treatments may be performed in an appropriately controlled way (e.g., case 4, Latin square design). For the morphed visual stimuli probe, conduct the experiment as in Step 10A(ii) by using stimuli that are morphed (or blended) versions of those used in Step 10A(ii). Transient treatments may be performed in an appropriately controlled way (e.g., case 4, Latin square design). For visual discrimination retention, rebaseline all subjects together when they have all reached criterion (at least) once, and then begin a retention interval (e.g., 7-10 d; exactly the same for each subject). Next, test the animals as in Step 10A(ii). It may be sufficient to test for a certain number of sessions to assess retention rather than testing until criterion is reattained. For reversal, reverse the reward contingencies, i.e., $\mathrm{S}^{+}$becomes $\mathrm{S}^{-}$and vice versa. For details, see ref. 5.

(v) Data analysis. Analyze performance measures for each visual discrimination phase (see Experimental design): number of sessions required to complete pretraining (Steps 5-9), and/or individual pretraining steps; percentage accuracy (in the form of an acquisition curve, if all subjects complete a certain minimum number of sessions, e.g., five or ten); sessions, trials and errors required to reach criterion; average correct and incorrect response latency; average magazine latency; percentage of bias (particularly in the first session for each animal); and perseveration score.

\section{(B) Object-location paired-associates learning}

(i) Object-location paired-associates learning training. Begin training on once-daily sessions of object-location pairedassociates learning, 5-7 d per week. Provide a single free reward (if your program does not do this automatically). Set up the apparatus as detailed for this task in MATERIALS and set up the software program for this stage with settings as detailed in Experimental design. Place each subject in its assigned chamber and start the session. Finish the session either after 60 min or after 90 trials (rat) or 36 trials (mouse) are completed (whichever comes first). After session termination, return each animal to its respective home cage.

$\triangle$ CRITICAL STEP Given that performance is likely to be poor at the start of training, with animals thus receiving many CTs per trial, limit sessions to 45 trials (rat) or 18 trials (mouse) in $60 \mathrm{~min}$. Continue until subjects can complete this in 30 min. Give each subject an even number of these reduced sessions, such that they can be combined into full 90- or 36-trial sessions for analysis. If the subject completes fewer trials than required, the missed trials may be added to the trials required in the next session (if fewer than $\sim 10$ ), or given in a new session. When you give a reduced number of trials per session, ensure that an equal number of each trial type is presented. 
$\triangle$ CRITICAL STEP To test the effects of a manipulation conducted before onset of the experiment or task acquisition (cases 1 and 2, see Experimental design), ensure that animals in the experimental and control groups complete comparable numbers of trials per session throughout task acquisition. Cap the number of trials given per session to accommodate the lowest responders.

$\Delta$ CRITICAL STEP At the end of each session, record the most crucial data for each subject (e.g., number of correct responses, number of trials completed), in case of computer malfunction. However, most software programs will record many other measures (see Data analysis).

(ii) Continue training on once-daily sessions of object-location paired-associates learning, 5-7 d per week until animals have reached the acquisition criterion for this task, at which point proceed to the next step. The acquisition criterion for this task is the completion of all trials with an accuracy of $80 \%$ (excluding CTs) for two consecutive sessions. If you are testing animals that received experimental manipulations before task acquisition (cases 1 and 2, see Experimental design), the main experimental readouts will probably be differences in the rates of acquisition (and/or final performance levels). Therefore, continue training all animals on all sessions, either for a given number of sessions (to allow plotting of an acquisition curve) or until the control and/or experimental group(s) attain criterion or stable performance. All animals may then progress to the same-object probe (Step 10B(iii); if required) on the same day. Alternatively, when postacquisition manipulations are to be conducted (e.g., cases 3 and 4, in combination with continued training at asymptotic performance level, see Experimental design), we suggest 'resting' animals when they reach criterion, with reminder sessions, until the entire group has achieved criterion. At that point, the entire group may be rebaselined before progressing to Step 10B(iii) (see Experimental design for details and alternatives). If subjects are scheduled to receive experimental treatments after acquisition but before Step 10B(iii) (case 3, see Experimental design), perform these when all animals have reached criterion (at least) once, making sure to counterbalance control and experimental groups according to acquisition performance. Rebaseline the subjects. $\Delta$ CRITICAL STEP In the case of microinfusion studies, animals should be rebaselined after surgery until a stable performance level is reached. Before commencing subsequent vehicle and drug infusion, a mock infusion involving the insertion of the infusion cannula only should be performed, followed by a vehicle infusion to assess nonspecific effects on performance.

$\Delta$ CRITICAL STEP The performance of mice on this task is less reliable than that of rats $8,45,54,56,60$, and it will depend on strain, age and so on. It may be necessary to apply a less-strict performance criterion (e.g., 70\%) for some strains of mice.

(iii) Post-training experimental manipulations. Depending on the aims of the experiment, perform appropriate post-training manipulations. Various post-training manipulations are possible. For continued training at the asymptotic performance level, conduct the experiment as in Step 10B(i). Transient treatments may be performed in an appropriately controlled way (e.g., case 4, Latin square design). The same-object probe may be conducted subsequently, if required. For the same-object probe, proceed as in Step 10B(i), but use a modified software program (as detailed in Experimental Design). Test animals for two sessions (once daily) of the same-object probe.

$\triangle$ CRITICAL STEP Avoid running the same-object probe before the animals have reached criterion, as exposure to the probe at this stage may encourage a visuomotor conditioning response (e.g., 'see object 1 in any location, respond to location $1^{\prime}$ ) rather than the formation of an object-location association.

(iv) Data analysis. Analyze the following behavioral variables (across acquisition, at performance asymptote and/or during the same-object probe, as appropriate for your study): number of sessions required to complete pretraining (Steps 5-9), and/or individual pretraining steps; percentage accuracy (in the form of an acquisition curve, if all subjects complete a certain minimum number of sessions, e.g., 30); sessions, trials and errors required to reach criterion; average correct and incorrect response latency; average magazine latency; percentage of bias; perseveration score; and trial type analysis (percentage accuracy for each of the six trial types individually).

\section{(C) VMCL}

(i) VMCL-specific pretraining. Provide a single free reward (if your program does not do this automatically). Set up the apparatus as detailed for this task in MATERIALS and set up the software program for this stage with settings as detailed in Experimental design. Place each subject in its assigned chamber and start the session. The session usually finishes either after $60 \mathrm{~min}$ or after 100 trials are completed (whichever comes first). After session termination, return each animal to its home cage. Continue training each animal once daily, 5-7 d per week, until it reaches a criterion of at least $80 \%$ of (noncorrection) trials correct (and all trials completed) in two consecutive sessions, with a LH of $2 \mathrm{~s}$. There is likely to be little variation in the number of days that animals require to complete VMCL-specific pretraining, but if there is a difference of 2 or more days between the fastest and slowest subjects, we suggest resting animals when they reach criterion, with reminder sessions, and then rebaselining the group so that the entire group can advance to VMCL training on the same day (see Experimental design). If subjects are scheduled to receive experimental treatments after pretraining but before task acquisition (case 2, see Experimental design), these may be performed now (instead of after Step 9), taking care to counterbalance control and experimental groups according to the number 
of sessions required to complete VMCL-specific pretraining. Rebaseline the subjects before task-specific training. $\triangle$ CRITICAL STEP At the end of each session, record crucial data for each subject (e.g., number of correct responses, number of trials completed) in case of computer malfunction. However, most software programs will record many other measures (see Data analysis).

$\triangle$ CRITICAL STEP If individual subjects have difficulty with the 2-s LH, use a longer LH (e.g., $5 \mathrm{~s}$ ) in the first instance, and then gradually reduce it as appropriate (on the basis of the subject's reaction time).

$\triangle$ CRITICAL STEP To test the effects of a manipulation conducted before onset of the experiment or task acquisition (e.g., case 1, see Experimental design), ensure that animals in experimental and control groups complete comparable numbers of trials per session throughout VMCL-specific pretraining. Cap the number of trials given per session to accommodate the lowest responders.

(ii) VMCL training. When all subjects have completed Step $10 \mathrm{C}(\mathrm{i})$, assign animals (of each experimental condition) to two groups counterbalanced according to the number of sessions required to achieve criterion for Step 10C(i). For one group, stimulus A will indicate that a response to the left location is correct (and right incorrect), and stimulus B will indicate the opposite. These contingencies will be reversed for the other group.

(iii) Begin VMCL training trials. Provide a single free reward (if your program does not do this automatically). Set up the apparatus as detailed for this task in MATERIALS and set up the software program for this stage with settings as detailed in Experimental design, with reward contingencies as appropriate for each subject. Place each subject in its assigned chamber and start the session. The session usually finishes either after 60 min or after 100 trials are completed (whichever comes first). After session termination, return each animal to its respective home cage. Train the animals once daily, 5-7 d per week.

$\triangle$ CRITICAL STEP Given that performance is likely to be poor at the start of training, with animals thus receiving many CTs per trial, limit sessions to 50 trials in 60 min for at least two sessions. Continue until subjects can complete this in $30 \mathrm{~min}$. Give each subject an even number of these reduced sessions, such that they can be combined into full 100-trial sessions for analysis. If the subject completes fewer trials than required, the missed trials may be added to the trials required in the next session (if fewer than 10 ) or given in a new session.

$\Delta$ CRITICAL STEP When testing the effects of a manipulation conducted before onset of the experiment or task acquisition (cases 1 and 2, see Experimental design), ensure that the animals in the experimental and control groups complete comparable numbers of trials per session throughout VMCL acquisition. Cap the number of trials given per session to accommodate the lowest responders.

$\Delta$ CRITICAL STEP Analyze the data after the first session, ensuring that no subject has a significant side bias (see Data analysis, Step $10 \mathrm{C}(\mathrm{vi})$ ).

(iv) Continue training on once-daily sessions of VMCL, 5-7 d per week, until animals have reached the acquisition criterion for this task, at which point you may proceed to the next step. The acquisition criterion for this task is the completion of all trials with an accuracy of $85 \%$ (excluding CTs) for two consecutive sessions. If you are testing animals that received experimental manipulations before task acquisition (cases 1 and 2, see Experimental design), the main experimental readouts will likely be differences in the rates of acquisition (and/or final performance levels). If so, continue training all animals on all sessions, either for a given number of sessions (to allow plotting of an acquisition curve) or until the control and/or experimental group(s) attain criterion or stable performance. Alternatively, if posttraining behavioral challenges (i.e., reversal, Step $10 \mathrm{C}(\mathrm{v})$ ) are of interest, and/or when postacquisition manipulations are to be conducted (e.g. cases 3 and 4, in combination with continued training at asymptotic performance level, see Experimental design), we suggest resting animals when they reach criterion, with reminder sessions, until the entire group has achieved criterion. At that point, the entire group may be rebaselined before progressing to Step $10 \mathrm{C}(\mathrm{v})$ (see Experimental design for details and alternatives). If subjects are scheduled to receive experimental treatments after acquisition but before Step $10 \mathrm{C}(\mathrm{v})$ (case 3, see Experimental design), perform these when all animals have reached criterion (at least) once, making sure to counterbalance control and experimental groups according to acquisition performance. Rebaseline the animals.

$\triangle$ CRITICAL STEP In the case of microinfusion studies, animals should be rebaselined until a stable performance level is reached after surgery. Before commencing subsequent vehicle and drug infusion, a mock infusion involving the insertion of the infusion cannula only should be performed; this should be followed by a vehicle infusion to assess nonspecific effects on performance.

(v) Post-training experimental manipulations. Depending on the aims of the experiment, perform appropriate post-training manipulations. Various post-training manipulations are possible. For continued training at the asymptotic performance level, conduct the experiment as in Step 10C(iii). Transient treatments may be performed in an appropriately controlled way (e.g., case 4, Latin square design). For reversal, proceed as in Step 10C(iii), but use modifications to the program. Continue training either until subjects reattain criterion ( $85 \%$ of trials correct on 2 consecutive days) or for a fixed number of sessions. 
(vi) Data analysis. Analyze performance measures for each VMCL phase (see Experimental design): number of sessions required to complete pretraining (Steps 5-9), and/or individual pretraining steps; percentage accuracy (in the form of an acquisition curve, if all subjects complete a certain minimum number of sessions); average correct and incorrect response latency; average magazine latency; percentage of bias (particularly in the first session for each animal); perseveration score and number of sessions, trials and errors required to reach criterion. Note that errors may be split into those committed when the animal is performing below chance, at chance and/or above chance, as appropriate.

\section{(D) Autoshaping}

(i) Autoshaping pretraining. Begin testing all subjects on the same day. Set up the apparatus as detailed for this task in MATERIALS and set up the software program for this stage with settings as detailed in Experimental design. Place each subject in its assigned chamber and start the session. Finish the session either after 60 min or after 40 trials are completed (whichever comes first). After session termination, return each animal to its respective home cage. Train subjects once daily, 5-7 d per week. Criterion for this stage is completing all trials in the allotted time, with all rewards consumed. There is likely to be little variation in the number of days that the animals require to complete this pretraining, but if there is a difference of 3 or more days between the fastest and slowest subjects we suggest resting animals when they reach criterion, with reminder sessions (see Experimental design for details and alternatives). The group may then be rebaselined together before advancing to autoshaping training on the same day. If subjects are scheduled to receive experimental treatments after pretraining but before task acquisition (case 2, see Experimental design), perform these now, making sure to counterbalance control and experimental groups according to the number of sessions required to complete pretraining. Rebaseline the subjects before task-specific training.

$\triangle$ CRITICAL STEP The autoshaping process may leave the animals with a side bias, even if the omission probe step is run. Therefore, we do not recommend that animals be tested on any other task after autoshaping. Animals need not necessarily be naive for this task, but we recommend that they are, because the associations formed in instrumental touchscreen tasks could possibly interfere with autoshaping.

$\triangle$ CRITICAL STEP At the end of each session, record critical data for each subject (e.g., number of trials completed, number of approaches to the left and right sides) in case of computer malfunction. However, most software programs will record many other measures (see Data analysis).

$\triangle$ CRITICAL STEP If you are testing the effects of a manipulation conducted before the onset of the experiment (case 1 , see Experimental design), ensure that the animals in the experimental and control groups complete comparable numbers of trials per session. Cap the number of trials given per session to accommodate the lowest responders.

(ii) Autoshaping acquisition training. Divide the animals (of each experimental condition) into ' $\mathrm{CS}+$ left side' and 'CS+ right side' groups. Counterbalance these according to the number of sessions required to complete Autoshaping pretraining (Step 10D(i)).

(iii) Set up the apparatus as detailed for this task in MATERIALS and set up the software program for this stage with settings as detailed in Experimental design, with reward contingencies as appropriate for each subject. Place each subject in its assigned chamber and start the session. The session finishes either after 90 min or after 40 trials are completed (whichever comes first). After session termination, return each animal to its respective home cage. Test all subjects for a minimum of two sessions, once daily, 5-7 d per week.

$\triangle$ CRITICAL STEP Data should be analyzed on a daily basis to monitor performance (see Data analysis, Step 10D(vi)). Animals should begin to discriminate between the $\mathrm{CS}^{+}$and $\mathrm{CS}^{-}$, as measured by the number of approaches to the two stimulus locations, within $\sim-5$ sessions. The latency to approach each location upon stimulus display should also indicate discriminative performance.

$\triangle$ CRITICAL STEP If you test the effects of a manipulation conducted before onset of the experiment or task acquisition (cases 1 and 2, see Experimental design), ensure that the animals in the experimental and control groups complete comparable numbers of trials per session throughout task acquisition. Cap the number of trials given per session to accommodate the lowest responders.

(iv) Continue training all subjects until the discriminated approach is clearly evident in the control group, regardless of experimental manipulation (cases 1-4, see Experimental design). If you are testing animals that received experimental manipulations before task acquisition (cases 1 and 2, see Experimental design), the main experimental readouts will likely be differences in the rates of acquisition (and/or final performance levels). If subjects are scheduled to receive experimental treatments after acquisition but before Step $10 \mathrm{D}(\mathrm{v})$ (e.g., case 3, in combination with continued training at asymptotic performance level, see Experimental design), perform these now, making sure to counterbalance control and experimental groups according to acquisition performance. Before progressing to Step $10 D(v)$, rebaseline all animals on acquisition training (see Experimental design) until the performance of all subjects has been stable for at least $2 \mathrm{~d}$. $\triangle$ CRITICAL STEP In microinfusion studies, animals should be rebaselined until a stable performance level is reached after surgery. Before commencing subsequent vehicle and drug infusion, a mock infusion involving the insertion of the infusion cannula only should be performed; this should be followed by a vehicle infusion to assess nonspecific effects on performance. 
(v) Post-training experimental manipulations. Depending on the aims of the experiment, perform appropriate post-training manipulations. Various post-training manipulations are possible. For continued training at the asymptotic performance level, conduct the experiment as in Step 10D(iii). Transient treatments may be performed in an appropriately controlled way (e.g., case 4, Latin square design). For the autoshaping omission probe, begin testing all subjects in the same session, on the day after their last autoshaping session. Set up the apparatus as detailed for this task in MATERIALS and set up the software program for this stage with settings as detailed in Experimental design. Place each subject in its assigned chamber and start the session. Finish the session either after 90 min or after 40 trials are completed (whichever comes first). After session termination, return each animal to its respective home cage. Test all subjects for a minimum of two sessions, once daily.

(vi) Data analysis. Analyze performance measures from autoshaping acquisition and omission sessions (see Experimental design): number of sessions required to complete pretraining (Step 5) and Autoshaping pretraining (Step 10D(i)); number of approaches made to the $\mathrm{CS}^{+}$and $\mathrm{CS}^{-}$when displayed; latency to approach each stimulus after onset; number and latency of touches to each stimulus after onset; and latency to enter the magazine upon reward delivery.

\section{? TROUBLESHOOTING}

\section{? TROUBLESHOOTING}

Consider excluding animals that fail to complete pretraining within a reasonable time frame (which may be determined ad hoc from the typical group performance). A dropout rate of $<10 \%$ is expected overall.

General troubleshooting advice can be found in Table 2. It is good practice to have spare light bulbs, touchscreen connector cables, IR beam assemblies, touchscreens, pump tubing and pellet dispensers available, because these components are particularly susceptible to failure. It is also important to check each test chamber at least once a week to ensure that IR beams, light stimuli and reward dispensers are functioning reliably. Campden software includes programs that may be used to check the function of these basic components at the start of each day.

TABLE 2 | Troubleshooting table.

\begin{tabular}{|c|c|c|}
\hline Problem & Possible reason & Solution \\
\hline \multirow{2}{*}{$\begin{array}{l}\text { Incomplete consumption of } \\
\text { reward }\end{array}$} & Animal insufficiently food restricted & Decrease weight as regulations permit \\
\hline & $\begin{array}{l}\text { Animal insufficiently habituated to } \\
\text { reward }\end{array}$ & Provide reward in home cage for additional days \\
\hline \multirow{2}{*}{ Unstable or poor performance } & Aversion to mask or touchscreen & $\begin{array}{l}\text { Increase exploration of the mask and screen by applying a } \\
\text { food reward to the mask (e.g., peanut butter, pellets or other) }\end{array}$ \\
\hline & Excessive fighting in the home cage & $\begin{array}{l}\text { Monitor the home cage and the general health of the animal, } \\
\text { separate animals if necessary }\end{array}$ \\
\hline \multirow[t]{5}{*}{$\begin{array}{l}\text { Abrupt decline in performance } \\
\text { and/or trial completion }\end{array}$} & $\begin{array}{l}\text { Touchscreen error (e.g., nonresponsive- } \\
\text { ness, not displaying images) }\end{array}$ & $\begin{array}{l}\text { Check physical connections, clean, run test program } \\
\text { (if available), recalibrate, reboot the system }\end{array}$ \\
\hline & $\begin{array}{l}\text { Reward delivery has ceased or } \\
\text { is inconsistent }\end{array}$ & $\begin{array}{l}\text { Check for physical blockage/disconnection, check for interface } \\
\text { error, replace }\end{array}$ \\
\hline & Initiation not detected & $\begin{array}{l}\text { Clean magazine photobeam, check physical connections, } \\
\text { replace if faulty }\end{array}$ \\
\hline & & For autoshaping: clean and test rear infrared beams \\
\hline & $\begin{array}{l}\text { Controlling system error (software or } \\
\text { hardware) }\end{array}$ & $\begin{array}{l}\text { Check physical connections, reboot the system, change } \\
\text { hardware if necessary }\end{array}$ \\
\hline
\end{tabular}




\section{TIMING}

Approximate timing for each step below is indicated as a number of sessions (i.e., days). As a rule, allow up to $\sim 80$ min per day per testing session from Step 5 onward (or 110 min for Step 10D(iii-v)). These 80 or 110 min include 60 (or 90) min of testing time, plus an additional 20 min for transporting animals from their home rooms to the testing room, setting up software and so on. Cumulative time taken to test all animals in an experiment depends on the capacity to load multiple animals per test run (i.e., number of chambers). Subsequent values for the number of days (sessions) it takes to execute these experiments typically reflect the approximate time it takes to test an average cohort of animals on each Step and are estimates based on our experience.

Steps 1-4, preparation for pretraining: $\sim 6$ or $10 \mathrm{~d}$. Timing depends on whether animals are acquired from an external source, in which case a 7-d acclimatization period is required before the onset of food restriction. After acclimatization, allow for $\sim 3 \mathrm{~d}$ of initial food restriction before the start of stage 1 pretraining. Regular handling and weighing of animals can be started $\sim 2 \mathrm{~d}$ after arrival. Reserve an average time per animal per day of $\sim 5 \mathrm{~min}$.

Steps 5-9, pretraining: 10-15 sessions. Note that pretraining may take longer (e.g., $\sim 10-30$ sessions) when a mask with small response windows (e.g., less than $3.0 \times 3.0 \mathrm{~cm}$ ) is used and/or if rebaselining is necessary. Also note that full pretraining is only necessary before the first instrumental task on which an animal is tested. Before subsequent instrumental tasks, animals should usually be tested on Step 9 only and, being well-trained, they may progress from this after only a few sessions.

Step $10 \mathrm{~A}(\mathrm{i}-\mathrm{iii})$, visual discrimination acquisition: the average number of sessions required to reach acquisition criterion with standard stimuli (Fig. 3a-c) is 5-6 (rat)/8-10 (mouse). Note that additional sessions may be required if resting and rebaselining are necessary (e.g., before Step $10 \mathrm{~A}(\mathrm{iv})$ ).

Step 10A(iv), visual discrimination post-training manipulations: the duration will depend on many factors, including experimental manipulation and performance. For retention, testing all animals for a predefined number of days (e.g., 5, 10) is likely to be sufficient.

Step 10B $(\mathrm{i}, \mathrm{ii})$, object-location paired-associated learning: as discussed in ANTICIPATED RESULTS, rats require an average of $\sim 34$ sessions to attain criterion of $80 \%$ correct, and mice need $\sim 50$ sessions to attain a less stringent criterion (70\%). Note that additional sessions may be required if resting and rebaselining are necessary (e.g., before Step 10B(iii)). Step 10B(iii), object-location paired associates learning same-object probe: two sessions Step $10 C(i)$, VMCL-specific pretraining: about two sessions, depending on performance Step 10C(ii-iv), VMCL training: about eight sessions; see ANTICIPATED RESULTS Step $10 \mathrm{D}(\mathrm{i})$, autoshaping pretraining: about two sessions

Step 10D(ii-iv), autoshaping acquisition training: four or five sessions, until discrimination is clearly evident in the control group

Step $10 \mathrm{D}(\mathrm{v})$, autoshaping omission probe: about two sessions

\section{ANTICIPATED RESULTS \\ Visual discrimination}

Figure 11 shows a typical visual discrimination acquisition curve of Lister hooded rats with photographic stimuli (Fig. 3d) (C.A.0., unpublished data). These data are available in Supplementary Data 1.

Object-location paired-associates learning The typical results presented in this section are based on the performance of male Lister hooded rats ( $n=24$; C.A.0., T.J.B., L.M.S., unpublished data) and male C57BL/6 mice on the object-location paired-associates learning task $8,45,54,56,60$, with data presented as means \pm s.e.m. Rats require an average of 2,220 \pm 184 trials or $34 \pm 1.9$ sessions to reach criterion ( $80 \%$ correct in two consecutive sessions). Mice require $\sim 50$ sessions (of 36 trials) to attain the less-stringent criterion (70\% correct in 2 consecutive days). Given the complexity of the task, it may occasionally (e.g., $\sim 1$ in 24 rats) be necessary to exclude, on an ad hoc basis, poor performers that are statistical outliers. At maximum performance level (i.e., after all animals have reached $80 \%$ criterion), animals perform as follows, on an average example session: percentage correct, $84.0 \pm 1.0$ (for rats; mice subjected to systemic saline treatment, $n=9: \sim 80-85 \%$ ) ${ }^{45}$; number of CTs, $19.5 \pm 1.5$ (mice: $9.55 \pm 4.44$ ); response latency to correct trials, $2.0 \pm 0.10 \mathrm{~s}$ (mice: $6.38 \pm 4.25 \mathrm{~s}$ ); response latency to incorrect trials, $2.1 \pm 0.13 \mathrm{~s}$ (mice: $7.20 \pm 4.35 \mathrm{~s}$ ); and average reward collection time, $2.36 \pm 0.04 \mathrm{~s}$ (mice: $2.36 \pm$ $1.07 \mathrm{~s})^{45}$. As for separate trial type analysis, we find that performance on particular trial types can be different for each animal, possibly depending on individual biases. Our rat task development data show that with the improved stimuli (Fig. 5b) there is little initial overall bias toward particular trial types, with average performance across rats on the first day of training ranging from $41.5 \pm 3.5 \%$ to $54.6 \pm 3.9 \%$. In control animals, transfer to the same-object probe (in our hands) does not lead to a change in performance level (rats: standard task: $84.0 \pm 1.0 \%$ to probe $82.6 \pm 1.7 \%$ ), indicating that it is unlikely that animals rely on a configural strategy. Compared with rats, mice demonstrate (on average) minimal variation in performance of the six standard 'flower-plane-spider' trial types (A.E.H., L.M.S. and T.J.B., unpublished data). 


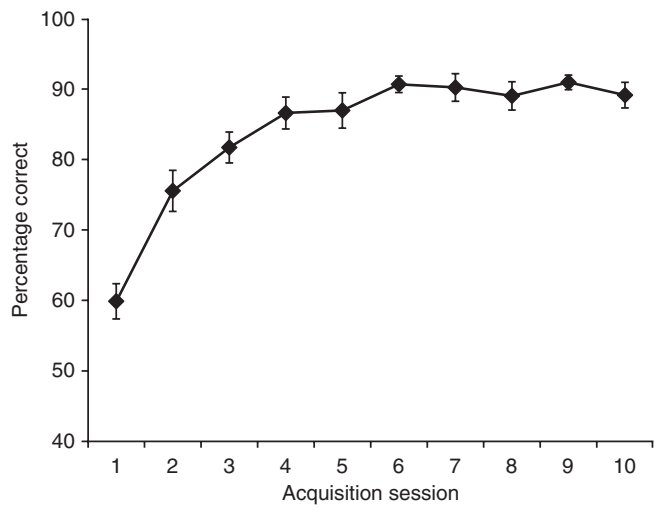

Figure 11 | Visual discrimination acquisition. Data shown are from 10-month-old sham-lesioned control rats ( $n=10$, with a history of PAL and TUNL) using photographic stimuli (C.A.0., unpublished results). Data are presented as mean \pm s.e.m.

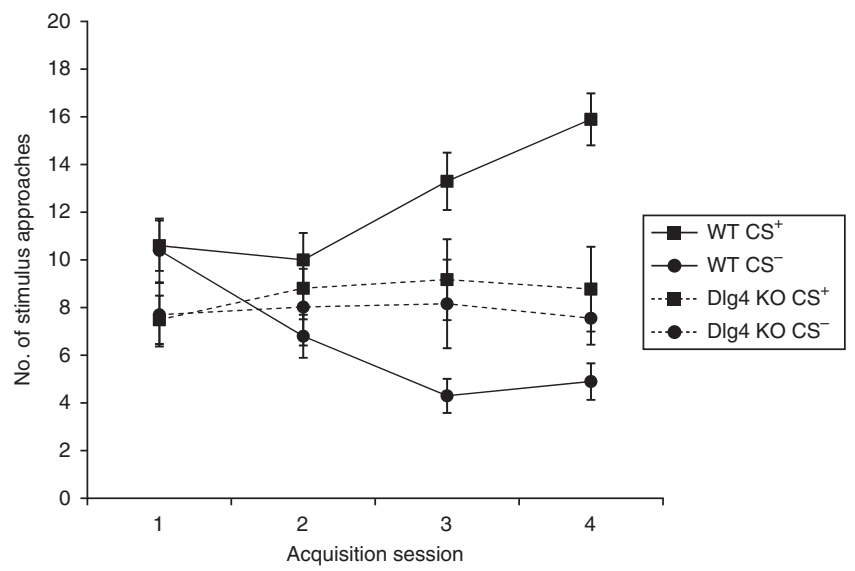

Figure 12 | Data from the autoshaping task. Number of approaches made by WT and Dlg4-/- mice $(n=10-15)$ to the $\mathrm{CS}^{+}$and $\mathrm{CS}^{-}$over four acquisition sessions ${ }^{8}$. Data are presented as mean \pm s.e.m. Adapted with permission from ref. 8.

VMCL

Sham-lesioned rats tested in accordance with published versions of this protocol (without the VMCL-specific pretraining phase described here) took, on average, fewer than 10 sessions (of 60 trials) to reach criterion ( $85 \%$ of trials correct on 2 consecutive days) for VMCL acquisition ${ }^{74}$. By using a near-identical reversal protocol to that presented here, sham-lesioned rats required, on average, 10 sessions (of 60 trials) to reach criterion ( $85 \%$ of trials correct on 2 consecutive days) for VMCL reversal ${ }^{74}$.

\section{Autoshaping}

Figure 12 shows the performance of $D\left(g 4^{-/-}\right.$and wild-type (WT) mice on the autoshaping task, as measured by the number of approaches to $\mathrm{CS}^{+}$and $\mathrm{CS}^{-}$. Stimulus discrimination rapidly developed in WT animals, with $\mathrm{CS}^{+}$approaches increasing and CS- $^{-}$approaches decreasing over four sessions ${ }^{8}$. Discrimination did not occur in the Dlg4 ${ }^{-/-}$group ${ }^{8}$. In the WT group, the latency to approach the $\mathrm{CS}^{+}$upon presentation also decreased between the first and fourth acquisition session, with no change in $\mathrm{CS}^{-}$approach latency ${ }^{8}$. No changes in approach latency to either stimulus were observed in the $D \lg ^{-1 /-}$ group $^{8}$ (latency data not shown).

To date, our laboratory has not used the autoshaping task with rats in the Campden apparatus. However, on the basis of the similarity of performance of rats and mice in this task (regardless of apparatus), we believe that the timing of the various stages and the resultant data should not differ substantially from those presented here.

With regard to the reward omission probe, previous experiments in rats have shown that although the total number of stimulus approaches decreases, the elevated number of $\mathrm{CS}^{+}$approaches, relative to $\mathrm{CS}^{-}$approaches, is maintained ${ }^{22}$. This is consistent with the Pavlovian nature of the stimulus-reward association learned in the Autoshaping task.

Note: Any Supplementary Information and Source Data files are available in the online version of the paper.

ACKNOWLEDGMENTS The protocols described here are those that are currently used in our laboratory, and they were written by current members of the group. However, many researchers have contributed to the development of touchscreen tasks, and we gratefully acknowledge their contribution. They include S. Bartko, J. Brigman, S. Forwood, C. Graybeal, A. Izquierdo, L. Lyon, A. Marti, K. McAllister, S. McTighe, J. Nithianantharajah, C. Romberg, J. Talpos and B. Winters. The research leading to these results has received support from the Innovative Medicine Initiative Joint Undertaking under grant agreement no. 115008, of which resources are composed of a European Federation of Pharmaceutical Industries and Associations in-kind contribution and financial contribution from the European Union's Seventh Framework Programme (FP7/2007-2013); the Wellcome Trust/Medical Research Council (089703/Z/09/Z) and Alzheimer's Research UK (ART/PG2006/5). A.E.H. receives funding from the European Union Seventh Framework Programme under grant agreement nos. 241995 (Project 'GENCODYS') and 242167 (Project 'SYNSYS'). J.A. was supported by the Swedish Academy of Pharmaceutical Sciences. A.H. was supported by the NIAAA Intramural Research Program.
AUTHOR CONTRIBUTIONS All authors contributed to the writing of this manuscript. A.E.H. coordinated this effort.

COMPETING FINANCIAL INTERESTS The authors declare competing financial interests: details are available in the online version of the paper.

Reprints and permissions information is available online at http://www.nature. com/reprints/index.html.

1. Bussey, T.J. et al. New translational assays for preclinical modelling of cognition in schizophrenia: The touchscreen testing method for mice and rats. Neuropharmacology 62, 1191-1203 (2012).

2. Bussey, T.J., Muir, J.L. \& Robbins, T.W. A novel automated touchscreen procedure for assessing learning in the rat using computer graphic stimuli. Neurosci. Res. Commun. 15, 103-110 (1994).

3. Bussey, T.J. et al. The touchscreen cognitive testing method for rodents: how to get the best out of your rat. Learn. Mem. 15, 516-523 (2008). 
4. Oomen, C.A. et al. The touchscreen operant platform for testing working memory and pattern separation in rats and mice. Nat. Protoc. 8, 2006-2021 (2013).

5. Mar, A.C. et al. The touchscreen operant platform for testing executive function in rats and mice. Nat. Protoc. 8, 1985-2005 (2013).

6. Romberg, C., Horner, A.E., Bussey, T.J. \& Saksida, L.M. A touch screenautomated cognitive test battery reveals impaired attention, memory abnormalities, and increased response inhibition in the TgCRND8 mouse model of Alzheimer's disease. Neurobiol. Aging 34, 731-744 (2013).

7. Romberg, C., Mattson, M.P., Mughal, M.R., Bussey, T.J. \& Saksida, L.M. Impaired attention in the $3 \times \operatorname{TgAD}$ mouse model of Alzheimer's disease: rescue by donepezil (Aricept). J. Neurosci. 31, 3500-3507 (2011).

8. Nithianantharajah, J. et al. Synaptic scaffold evolution generated components of vertebrate cognitive complexity. Nat. Neurosci. 16, 16-24 (2013).

9. Brigman, J.L., Ihne, J., Saksida, L.M., Bussey, T.J. \& Holmes, A. Effects of subchronic phencyclidine (PCP) treatment on social behaviors, and operant discrimination and reversal learning in $\mathrm{C} 57 \mathrm{BL} / 6 \mathrm{~J}$ mice. Front. Behav. Neurosci. 3, 2 (2009).

10. Brigman, J.L., Padukiewicz, K.E., Sutherland, M.L. \& Rothblat, L.A. Executive functions in the heterozygous reeler mouse model of schizophrenia. Behav. Neurosci. 120, 984-988 (2006).

11. Morton, A.J., Skillings, E., Bussey, T.J. \& Saksida, L.M. Measuring cognitive deficits in disabled mice using an automated interactive touchscreen system. Nat. Methods 3, 767 (2006).

12. Creer, D.J., Romberg, C., Saksida, L.M., van Praag, H. \& Bussey, T.J. Running enhances spatial pattern separation in mice. Proc. Natl. Acad. Sci. USA 107, 2367-2372 (2010).

13. Graybeal, C. et al. Paradoxical reversal learning enhancement by stress or prefrontal cortical damage: rescue with BDNF. Nat. Neurosci. 14, 1507-1509 (2011).

14. Izquierdo, A. et al. Reversal-specific learning impairments after a binge regimen of methamphetamine in rats: possible involvement of striatal dopamine. Neuropsychopharmacology 35, 505-514 (2010).

15. Aggleton, J.P., Keen, S., Warburton, E.C. \& Bussey, T.J. Extensive cytotoxic lesions involving both the rhinal cortices and area TE impair recognition but spare spatial alternation in the rat. Brain Res. Bull. 43, 279-287 (1997).

16. Bussey, T.J., Dias, R., Amin, E., Muir, J.L. \& Aggleton, J.P. Perirhinal cortex and place-object conditional learning in the rat. Behav. Neurosci. 115 , 776-785 (2001).

17. Bussey, T.J. et al. Intact negative patterning in rats with fornix or combined perirhinal and postrhinal cortex lesions. Exp. Brain Res. 134, 506-519 (2000).

18. Winters, B.D., Bartko, S.J., Saksida, L.M. \& Bussey, T.J. Muscimol, AP5, or scopolamine infused into perirhinal cortex impairs two-choice visual discrimination learning in rats. Neurobiol. Learn. Mem. 93, 221-228 (2010).

19. Brigman, J.L. \& Rothblat, L.A. Stimulus specific deficit on visual reversal learning after lesions of medial prefrontal cortex in the mouse. Behav. Brain Res. 187, 405-410 (2008).

20. Christakou, A., Robbins, T.W. \& Everitt, B.J. Functional disconnection of a prefrontal cortical-dorsal striatal system disrupts choice reaction time performance: implications for attentional function. Behav. Neurosci. 115, 812-825 (2001).

21. Christakou, A., Robbins, T.W. \& Everitt, B.J. Prolonged neglect following unilateral disruption of a prefrontal cortical-dorsal striatal system. Eur. J. Neurosci. 21, 782-792 (2005).

22. Bussey, T.J., Everitt, B.J. \& Robbins, T.W. Dissociable effects of cingulate and medial frontal cortex lesions on stimulus-reward learning using a novel Pavlovian autoshaping procedure for the rat: implications for the neurobiology of emotion. Behav. Neurosci. 111, 908-919 (1997).

23. Bussey, T.J., Muir, J.L., Everitt, B.J. \& Robbins, T.W. Triple dissociation of anterior cingulate, posterior cingulate, and medial frontal cortices on visual discrimination tasks using a touchscreen testing procedure for the rat. Behav. Neurosci. 111, 920-936 (1997).

24. Cardinal, R.N. et al. Role of the anterior cingulate cortex in the control over behavior by Pavlovian conditioned stimuli in rats. Behav. Neurosci. 117, 566-587 (2003).

25. Cardinal, R.N. et al. Effects of selective excitotoxic lesions of the nucleus accumbens core, anterior cingulate cortex, and central nucleus of the amygdala on autoshaping performance in rats. Behav. Neurosci. 116, 553-567 (2002).

26. Parkinson, J.A., Willoughby, P.J., Robbins, T.W. \& Everitt, B.J. Disconnection of the anterior cingulate cortex and nucleus accumbens core impairs Pavlovian approach behavior: further evidence for limbic cortical-ventral striatopallidal systems. Behav. Neurosci. 114, 42-63 (2000).
27. Abela, A.R. \& Chudasama, Y. Dissociable contributions of the ventral hippocampus and orbitofrontal cortex to decision-making with a delayed or uncertain outcome. Eur. J. Neurosci 37, 640-647 (2013).

28. Chudasama, Y. \& Robbins, T.W. Dissociable contributions of the orbitofrontal and infralimbic cortex to Pavlovian autoshaping and discrimination reversal learning: further evidence for the functional heterogeneity of the rodent frontal cortex. J. Neurosci. 23, 8771-8780 (2003).

29. Chudasama, Y. \& Muir, J.L. Visual attention in the rat: a role for the prelimbic cortex and thalamic nuclei? Behav. Neurosci. 115, 417-428 (2001).

30. Dalley, J.W. et al. Time-limited modulation of appetitive Pavlovian memory by $D 1$ and NMDA receptors in the nucleus accumbens. Proc. Natl. Acad. Sci. USA 102, 6189-6194 (2005).

31. Dalley, J.W. et al. Nucleus accumbens dopamine and discriminated approach learning: interactive effects of 6-hydroxydopamine lesions and systemic apomorphine administration. Psychopharmacology (Berl.) 161, 425-433 (2002).

32. Parkinson, J.A., Robbins, T.W. \& Everitt, B.J. Dissociable roles of the central and basolateral amygdala in appetitive emotional learning. Eur. J. Neurosci. 12, 405-413 (2000).

33. Winstanley, C.A., Baunez, C., Theobald, D.E. \& Robbins, T.W. Lesions to the subthalamic nucleus decrease impulsive choice but impair autoshaping in rats: the importance of the basal ganglia in Pavlovian conditioning and impulse control. Eur. J. Neurosci. 21, 3107-3116 (2005).

34. Bussey, T.J., Clea Warburton, E., Aggleton, J.P. \& Muir, J.L. Fornix lesions can facilitate acquisition of the transverse patterning task: a challenge for 'configural' theories of hippocampal function. J. Neurosci. 18, 1622-1631 (1998).

35. Abela, A.R., Dougherty, S.D., Fagen, E.D., Hill, C.J. \& Chudasama, Y. Inhibitory control deficits in rats with ventral hippocampal lesions. Cereb. Cortex 23, 1396-1409 (2013).

36. Ito, R., Everitt, B.J. \& Robbins, T.W. The hippocampus and appetitive Pavlovian conditioning: effects of excitotoxic hippocampal lesions on conditioned locomotor activity and autoshaping. Hippocampus 15, 713-721 (2005).

37. Kim, S., Lee, J. \& Lee, I. The hippocampus is required for visually cued contextual response selection, but not for visual discrimination of contexts. Front. Behav. Neurosci. 6, 66 (2012)

38. McTighe, S.M., Mar, A.C., Romberg, C., Bussey, T.J. \& Saksida, L.M. A new touchscreen test of pattern separation: effect of hippocampal lesions. Neuroreport 20, 881-885 (2009).

39. Talpos, J.C., Dias, R., Bussey, T.J. \& Saksida, L.M. Hippocampal lesions in rats impair learning and memory for locations on a touch-sensitive computer screen: the 'ASAT' task. Behav. Brain Res. 192, 216-225 (2008).

40. Talpos, J.C., McTighe, S.M., Dias, R., Saksida, L.M. \& Bussey, T.J. Trial-unique, delayed nonmatching-to-location (TUNL): a novel, highly hippocampus-dependent automated touchscreen test of location memory and pattern separation. Neurobiol. Learn. Mem. 94, 341-352 (2010).

41. Talpos, J.C., Winters, B.D., Dias, R., Saksida, L.M. \& Bussey, T.J. A novel touchscreen-automated paired-associate learning (PAL) task sensitive to pharmacological manipulation of the hippocampus: a translational rodent model of cognitive impairments in neurodegenerative disease. Psychopharmacology (Berl) 205, 157-168 (2009).

42. Inglis, W.L., Olmstead, M.C. \& Robbins, T.W. Pedunculopontine tegmental nucleus lesions impair stimulus--reward learning in autoshaping and conditioned reinforcement paradigms. Behav. Neurosci. 114, 285-294 (2000).

43. Janisiewicz, A.M. \& Baxter, M.G. Transfer effects and conditional learning in rats with selective lesions of medial septal/diagonal band cholinergic neurons. Behav. Neurosci. 117, 1342-1352 (2003).

44. Botly, L.C. \& De Rosa, E. Impaired visual search in rats reveals cholinergic contributions to feature binding in visuospatial attention. Cereb. Cortex 22, 2441-2453 (2012).

45. Bartko, S.J., Vendrell, I., Saksida, L.M. \& Bussey, T.J. A computerautomated touchscreen paired-associates learning (PAL) task for mice: impairments following administration of scopolamine or dicyclomine and improvements following donepezil. Psychopharmacology (Berl) 214, 537-548 (2011).

46. Chen, W.S., Wong, F.K., Chapman, P.F. \& Pemberton, D.J. Effect of donepezil on reversal learning in a touch screen-based operant task. Behav. Pharmacol. 20, 653-656 (2009).

47. McCarthy, A.D. et al. FK962 and donepezil act synergistically to improve cognition in rats: potential as an add-on therapy for Alzheimer's disease. Pharmacol. Biochem. Behav. 98, 76-80 (2011).

48. Talpos, J.C., Fletcher, A.C., Circelli, C., Tricklebank, M.D. \& Dix, S.L. The pharmacological sensitivity of a touchscreen-based visual discrimination task in the rat using simple and perceptually challenging stimuli. Psychopharmacology (Berl.) 221, 437-449 (2012). 
49. Izquierdo, A. et al. Genetic and dopaminergic modulation of reversal learning in a touchscreen-based operant procedure for mice. Behav. Brain Res. 171, 181-188 (2006).

50. Steckler, T. \& Sahgal, A. Psychopharmacological studies in rats responding at touch-sensitive devices. Psychopharmacology (Berl.) 118, 226-229 (1995).

51. Brigman, J.L. et al. Pharmacological or genetic inactivation of the serotonin transporter improves reversal learning in mice. Cereb. Cortex 20, 1955-1963 (2010).

52. Izquierdo, A. et al. Impaired reward learning and intact motivation after serotonin depletion in rats. Behav. Brain Res. 233, 494-499 (2012).

53. Winstanley, C.A., Dalley, J.W., Theobald, D.E. \& Robbins, T.W. Fractionating impulsivity: contrasting effects of central 5-HT depletion on different measures of impulsive behavior. Neuropsychopharmacology 29, 1331-1343 (2004).

54. Coba, M.P. et al. TNiK is required for postsynaptic and nuclear signaling pathways and cognitive function. J. Neurosci. 32, 13987-13999 (2012).

55. Karlsson, R.M. et al. Assessment of glutamate transporter GLAST (EAAT1)deficient mice for phenotypes relevant to the negative and executive/ cognitive symptoms of schizophrenia. Neuropsychopharmacology 34, 1578-1589 (2009)

56. Bartko, S.J. et al. Intact attentional processing but abnormal responding in $M_{1}$ muscarinic receptor-deficient mice using an automated touchscreen method. Neuropharmacology 61, 1366-1378 (2011).

57. Brigman, J.L. et al. Impaired discrimination learning in mice lacking the NMDA receptor NR2A subunit. Learn. Mem. 15, 50-54 (2008).

58. Barkus, C. et al. Do GluA1 knockout mice exhibit behavioral abnormalities relevant to the negative or cognitive symptoms of schizophrenia and schizoaffective disorder? Neuropharmacology 62, 1263-1272 (2012).

59. Ryan, T.J. et al. Evolution of GluN2A/B cytoplasmic domains diversified vertebrate synaptic plasticity and behavior. Nat. Neurosci. 16, 25-32 (2013).

60. Clelland, C.D. et al. A functional role for adult hippocampal neurogenesis in spatial pattern separation. Science 325, 210-213 (2009)

61. Minini, L. \& Jeffery, K.J. Do rats use shape to solve 'shape discriminations'? Learn. Mem. 13, 287-297 (2006).

62. Brigman, J.L. et al. GluN2B in corticostriatal circuits governs choice learning and choice shifting. Nat. Neurosci. 16, 1101-1110 (2013).

63. Bussey, T.J., Saksida, L.M. \& Rothblat, L.A. Discrimination of computergraphic stimuli by mice: a method for the behavioral characterization of transgenic and gene-knockout models. Behav. Neurosci. 115, 957-960 (2001).

64. Morrison, S.K. \& Brown, M.F. The touch screen system in the pigeon laboratory: an initial evaluation of its utility. Behav. Res. Methods Instrum. Comput. 22, 123-126 (1990).

65. Leising, K.J., Wolf, J.E. \& Ruprecht, C.M. Visual discrimination learning with an iPad-equipped apparatus. Behav. Process. 93, 140-147 (2013).

66. Roberts, A.C., Robbins, T.W. \& Everitt, B.J. The effects of intradimensional and extradimensional shifts on visual discrimination learning in humans and non-human primates. Q. J. Exp. Psychol. B 40, 321-341 (1988).

67. Gaffan, D. et al. Effects of fornix transection upon associative memory in monkeys: role of the hippocampus in learned action. Q. J. Exp. Psychol. B 36, 173-221 (1984).

68. Sahgal, A. \& Steckler, T. TouchWindows and operant behaviour in rats. J. Neurosci. Methods 55, 59-64 (1994).

69. Jones, B. \& Mishkin, M. Limbic lesions and the problem of stimulusreinforcement associations. Exp. Neurol. 36, 362-377 (1972).

70. Brigman, J.L., Bussey, T.J., Saksida, L.M. \& Rothblat, L.A. Discrimination of multidimensional visual stimuli by mice: intra- and extradimensional shifts. Behav. Neurosci. 119, 839-842 (2005).

71. Markham, M.R., Butt, A.E. \& Dougher, M.J. A computer touch-screen apparatus for training visual discriminations in rats. J. Exp. Anal. Behav. 65 173-182 (1996)

72. Bussey, T.J., Muir, J.L., Everitt, B.J. \& Robbins, T.W. Dissociable effects of anterior and posterior cingulate cortex lesions on the acquisition of a conditional visual discrimination: facilitation of early learning vs. impairment of late learning. Behav. Brain Res. 82, 45-56 (1996).

73. Muir, J.L., Bussey, T.J., Everitt, B.J. \& Robbins, T.W. Dissociable effects of AMPA-induced lesions of the vertical limb diagonal band of Broca on performance of the 5-choice serial reaction time task and on acquisition of a conditional visual discrimination. Behav. Brain Res. 82, 31-44 (1996).

74. Chudasama, Y., Bussey, T.J. \& Muir, J.L. Effects of selective thalamic and prelimbic cortex lesions on two types of visual discrimination and reversal learning. Eur. J. Neurosci. 14, 1009-1020 (2001).
75. Sahakian, B.J. et al. A comparative study of visuospatial memory and learning in Alzheimer-type dementia and Parkinson's disease. Brain 111, 695-718 (1988).

76. Stip, E. et al. Cognitive discernible factors between schizophrenia and schizoaffective disorder. Brain Cogn. 59, 292-295 (2005).

77. Barnett, J.H. et al. Assessing cognitive function in clinical trials of schizophrenia. Neurosci. Biobehav. Rev. 34, 1161-1177 (2010).

78. Barnett, J.H. et al. Visuospatial learning and executive function are independently impaired in first-episode psychosis. Psychol. Med. 35, 1031-1041 (2005).

79. Lange, K.W., Sahakian, B.J., Quinn, N.P., Marsden, C.D. \& Robbins, T.W. Comparison of executive and visuospatial memory function in Huntington's disease and dementia of Alzheimer type matched for degree of dementia. J. Neurol. Neurosurg. Psychiatry 58, 598-606 (1995).

80. Porter, R.J., Gallagher, P., Thompson, J.M. \& Young, A.H. Neurocognitive impairment in drug-free patients with major depressive disorder. Br. J. Psychiatry 182, 214-220 (2003).

81. Sweeney, J.A., Kmiec, J.A. \& Kupfer, D.J. Neuropsychologic impairments in bipolar and unipolar mood disorders on the CANTAB neurocognitive battery. Biol. Psychiatry 48, 674-684 (2000).

82. Sahakian, B.J. et al. Sparing of attentional relative to mnemonic function in a subgroup of patients with dementia of the Alzheimer type. Neuropsychologia 28, 1197-1213 (1990).

83. Sahgal, A. et al. Detection of visual memory and learning deficits in Alzheimer's disease using the Cambridge Neuropsychological Test Automated Battery. Dementia 2, 150-158 (1991).

84. Fowler, K.S., Saling, M.M., Conway, E.L., Semple, J.M. \& Louis, W.J. Computerized neuropsychological tests in the early detection of dementia: prospective findings. J. Int. Neuropsychol. Soc. 3, 139-146 (1997).

85. Blackwell, A.D. et al. Detecting dementia: novel neuropsychological markers of preclinical Alzheimer's disease. Dement. Geriatr. Cogn. Disord. 17, 42-48 (2004).

86. Swainson, R. et al. Early detection and differential diagnosis of Alzheimer's disease and depression with neuropsychological tasks. Dement. Geriatr. Cogn. Disord. 12, 265-280 (2001).

87. Milner, B., Johnsrude, I. \& Crane, J. Right medial temporal-lobe contribution to object-location memory. Philos. Trans. R. Soc. Lond. B Biol. Sci. 352, 1469-1474 (1997).

88. Owen, A.M., Sahakian, B.J., Semple, J., Polkey, C.E. \& Robbins, T.W. Visuo-spatial short-term recognition memory and learning after temporal lobe excisions, frontal lobe excisions or amygdalo-hippocampectomy in man. Neuropsychologia 33, 1-24 (1995).

89. Simons, J.S. \& Spiers, H.J. Prefrontal and medial temporal lobe interactions in long-term memory. Nat. Rev. Neurosci. 4, 637-648 (2003).

90. McAllister, K.A., Saksida, L.M. \& Bussey, T.J. Dissociation between memory retention across a delay and pattern separation following medial prefrontal cortex lesions in the touchscreen TUNL task. Neurobiol. Learn. Mem. 101 120-126 (2013).

91. Talpos, J.C. in Proceedings of Measuring Behavior 2012, $8^{\text {th }}$ International Conference on Methods and Techniques in Behavioral Research (Utrecht, The Netherlands, August 28-31, 2012) (eds. Spink, A.J. et al.)(Noldus Information Technology, 2012).

92. Murray, E.A., Bussey, T.J. \& Wise, S.P. Role of prefrontal cortex in a network for arbitrary visuomotor mapping. Exp. Brain Res. 133, 114-129 (2000).

93. Sziklas, V., Petrides, M. \& Leri, F. The effects of lesions to the mammillary region and the hippocampus on conditional associative learning by rats. Eur. J. Neurosci. 8, 106-115 (1996).

94. Brasted, P.J., Bussey, T.J., Murray, E.A. \& Wise, S.P. Role of the hippocampal system in associative learning beyond the spatial domain. Brain 126, 1202-1223 (2003).

95. Reading, P.J., Dunnett, S.B. \& Robbins, T.W. Dissociable roles of the ventral, medial and lateral striatum on the acquisition and performance of a complex visual stimulus-response habit. Behav. Brain Res. 45, 147-161 (1991).

96. Marston, H.M., Everitt, B.J. \& Robbins, T.W. Comparative effects of excitotoxic lesions of the hippocampus and septum/diagonal band on conditional visual discrimination and spatial learning. Neuropsychologia 31, 1099-1118 (1993).

97. Bussey, T.J., Duck, J., Muir, J.L. \& Aggleton, J.P. Distinct patterns of behavioural impairments resulting from fornix transection or neurotoxic lesions of the perirhinal and postrhinal cortices in the rat. Behav. Brain Res. 111, 187-202 (2000).

98. Hay, J.F., Moscovitch, M. \& Levine, B. Dissociating habit and recollection: evidence from Parkinson's disease, amnesia and focal lesion patients. Neuropsychologia 40, 1324-1334 (2002). 
99. Witt, K., Nuhsman, A. \& Deuschl, G. Dissociation of habit-learning in Parkinson's and cerebellar disease. J. Cogn. Neurosci. 14, 493-499 (2002).

100. Brown, P.L. \& Jenkins, H.M. Auto-shaping of the pigeon's key-peck. J. Exp. Anal. Behav. 11, 1-8 (1968).

101. Wilcove, W.G. \& Miller, J.C. CS-USC presentations and a lever: human autoshaping. J. Exp. Psychol. 103, 868-877 (1974).

102. Sidman, M. \& Fletcher, F.G. A demonstration of auto-shaping with monkeys. J. Exp. Anal. Behav. 11, 307-309 (1968).

103. Wasserman, E.A. Pavlovian conditioning with heat reinforcement produces stimulus-directed pecking in chicks. Science 181, 875-877 (1973).

104. Jenkins, H.M., Barrera, F.J., Ireland, C. \& Woodside, B. Signal-centered action patterns of dogs in appetitive classical conditioning. Learn. Motiv. 9, 272-296 (1978).

105. Stiers, M. \& Silberberg, A. Lever-contact responses in rats: automaintenance with and without a negative response-reinforcer dependency. J. Exp. Anal. Behav. 22, 497-506 (1974).

106. Cleland, G.G. \& Davey, G.C. Autoshaping in the rat: the effects of localizable visual and auditory signals for food. J. Exp. Anal. Behav. 40, 47-56 (1983).

107. Di Ciano, P., Cardinal, R.N., Cowell, R.A., Little, S.J. \& Everitt, B.J. Differential involvement of NMDA, AMPA/kainate, and dopamine receptors in the nucleus accumbens core in the acquisition and performance of Pavlovian approach behavior. J. Neurosci. 21, 9471-9477 (2001).

108. Williams, D.R. \& Williams, H. Auto-maintenance in the pigeon: sustained pecking despite contingent non-reinforcement. J. Exp. Anal. Behav. 12, 511-520 (1969).

109. Parkinson, J.A. et al. Nucleus accumbens dopamine depletion impairs both acquisition and performance of appetitive Pavlovian approach behaviour: implications for mesoaccumbens dopamine function. Behav. Brain Res. 137, 149-163 (2002).

110. Brog, J.S., Salyapongse, A., Deutch, A.Y. \& Zahm, D.S. The patterns of afferent innervation of the core and shell in the 'accumbens' part of the rat ventral striatum: immunohistochemical detection of retrogradely transported fluoro-gold. J. Comp. Neurol. 338, 255-278 (1993).

111. Danna, C.L. \& Elmer, G.I. Disruption of conditioned reward association by typical and atypical antipsychotics. Pharmacol. Biochem. Behav. 96, 40-47 (2010).

112. Coyle, J.T., Basu, A., Benneyworth, M., Balu, D. \& Konopaske, G. Glutamatergic synaptic dysregulation in schizophrenia: therapeutic implications. Handb. Exp. Pharmacol. 267-295 (2012).
113. Goto, Y. \& Grace, A.A. The dopamine system and the pathophysiology of schizophrenia: a basic science perspective. Int. Rev. Neurobiol. 78, 41-68 (2007).

114. Flagel, S.B., Watson, S.J., Robinson, T.E. \& Akil, H. Individual differences in the propensity to approach signals vs. goals promote different adaptations in the dopamine system of rats. Psychopharmacology (Berl.) 191, 599-607 (2007).

115. Flagel, S.B., Watson, S.J., Akil, H. \& Robinson, T.E. Individual differences in the attribution of incentive salience to a reward-related cue: influence on cocaine sensitization. Behav. Brain Res. 186, 48-56 (2008).

116. Muir, J.L. Attention and stimulus processing in the rat. Brain Res. Cogn. Brain Res. 3, 215-225 (1996).

117. Clark, R.E., Reinagel, P., Broadbent, N.J., Flister, E.D. \& Squire, L.R. Intact performance on feature-ambiguous discriminations in rats with lesions of the perirhinal cortex. Neuron 70, 132-140 (2011).

118. Cook, R.G., Geller, A.I., Zhang, G.R. \& Gowda, R. Touchscreen-enhanced visual learning in rats. Behav. Res. Methods Instrum. Comput. 36, 101-106 (2004).

119. Frick, K.M. \& Berger-Sweeney, J. Spatial reference memory and neocortical neurochemistry vary with the estrous cycle in $\mathrm{C57BL} / 6$ mice. Behav. Neurosci. 115, 229-237 (2001).

120. Meziane, H., Ouagazzal, A.M., Aubert, L., Wietrzych, M. \& Krezel, W. Estrous cycle effects on behavior of $[57 \mathrm{BL} / 6 \mathrm{~J}$ and BALB/cByJ female mice: implications for phenotyping strategies. Genes Brain Behav. 6, 192-200 (2007).

121. Lederle, L. et al. Reward-related behavioral paradigms for addiction research in the mouse: performance of common inbred strains. PLOS ONE 6, e15536 (2011).

122. Beeler, J.A., Prendergast, B. \& Zhuang, X. Low amplitude entrainment of mice and the impact of circadian phase on behavior tests. Physiol. Behav. 87, 870-880 (2006).

123. Roedel, A., Storch, C., Holsboer, F. \& Ohl, F. Effects of light or dark phase testing on behavioral and cognitive performance in DBA mice. Lab Anim. 40, 371-381 (2006).

124. Chaudhury, D. \& Colwell, C.S. Circadian modulation of learning and memory in fear-conditioned mice. Behav. Brain Res. 133, 95-108 (2002).

125. Satoh, Y., Kawai, H., Kudo, N., Kawashima, Y. \& Mitsumoto, A. Temperature rhythm reentrains faster than locomotor rhythm after a light phase shift. Physiol. Behav. 88, 404-410 (2006).

126. Cardinal, R.N. \& Aitken, M.R. Whisker: a client-server high-performance multimedia research control system. Behav. Res. Methods 42, 1059-1071 (2010). 